hep-th/0403051

MIT-CTP-3476

\title{
Twisted Tachyon Condensation in Closed String Field Theory
}

\author{
Yuji Okawa and Barton Zwiebach \\ Center for Theoretical Physics \\ Massachusetts Institute of Technology \\ Cambridge, MA 02139, USA \\ okawa@lns.mit.edu, zwiebach@lns.mit.edu
}

\begin{abstract}
We consider twisted tachyons on $\mathbb{C} / \mathbb{Z}_{N}$ orbifolds of bosonic closed string theory. It has been conjectured that these tachyonic instabilities correspond to decays of the orbifolds into flat space or into orbifolds with smaller deficit angles. We examine this conjecture using closed string field theory, with the string field truncated to low-level tachyons. We compute the tachyon potentials for $\mathbb{C} / \mathbb{Z}_{2}$ and $\mathbb{C} / \mathbb{Z}_{3}$ orbifolds and find critical points at depths that generate about $70 \%$ of the expected change in the deficit angle. We find that both twisted fields and untwisted modes localized near the apex of the cone acquire vacuum expectation values and contribute to the potential.
\end{abstract}




\section{Contents}

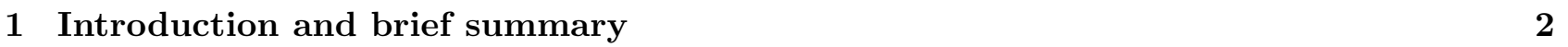

2 The conjecture and closed string field theorv

2.1 The coniecture and its refinement . . . . . . . . . . . . . . . . 5

2.2 Closed string field theorv and Newton's constant . . . . . . . . . . . . . . . 8

3 Orbifold CFT correlators 11

4 The $\mathbb{C} / \mathbb{Z}_{2}$ tachvon potential $\quad 15$

4.1 Computing the action . . . . . . . . . . . . . . . . . 16

4.2 A preliminarv analvsis . . . . . . . . . . . . . . . . . . . . . . 19

4.3 Level expansion analvsis . . . . . . . . . . . . . . . . . . . . . . . . . 20

4.3 .1 Coordinate space method . . . . . . . . . . . . . . . . . . 21

4.3.2 Momentum space method . . . . . . . . . . . . . . . . . . . 25

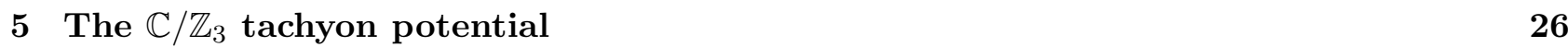

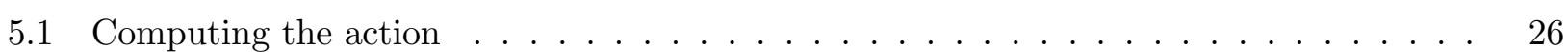

5.2 A preliminarv analysis . . . . . . . . . . . . . . . . . . 28

5.3 Level expansion analvsis . . . . . . . . . . . . . . . . . . . . . . . . . . . . . 29

6 Elementary four-point vertices $\quad 30$

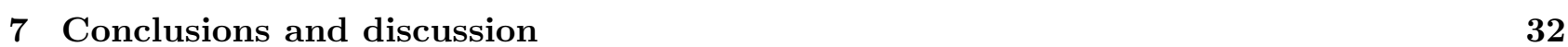

\section{Introduction and brief summary}

In a stimulating paper, Adams, Polchinski, and Silverstein [1 gave a physical interpretation of the instability associated with the twisted closed string tachyons of the $\mathbb{C} / \mathbb{Z}_{N}$ orbifold in Type II string theory. In this orbifold two spatial dimensions form a cone and twisted closed string states live at the apex of the cone - an eight-dimensional subspace of the ten-dimensional spacetime. It was conjectured in [1] that the instability signals the possible decay of the cone into flat two-dimensional space or into a cone with a smaller deficit angle. Tests of this conjecture were discussed in detail in 1, 2, 3, making use of probe D-branes and the tools of conformal field theory (CFT). Some aspects of the time-dependent decay process were examined in the supergravity limit 4, 5. The tachyons themselves do not feature in this analysis, which instead focuses on the radial propagation of gravity and dilaton waves on the cone. Presumably these waves are generated by the condensation of twisted tachyons at the apex. Various issues concerning twisted closed string tachyons have been discussed in [6, 7, 8, 9].

In this paper, we present a different approach to twisted tachyon condensation. We use closed string field theory (CSFT) on the $\mathbb{C} / \mathbb{Z}_{N}$ backgrounds to provide evidence for the conjecture in the framework 
of level truncation. The work of [1] considered Type II orbifolds $\mathbb{C} / \mathbb{Z}_{N}$ with odd $N$, where the bulk tachyon is absent. Since a covariant closed superstring field theory has not yet been constructed, we focus on bosonic strings, for which closed string field theory exists [10, 11, 12, 13. We believe that the interpretation for the instability applies to the bosonic string, despite the lack of supersymmetry and the presence of a bulk tachyon. Indeed, both the original orbifold $\mathbb{C} / \mathbb{Z}_{N}$ and a flat 2-space are consistent bosonic string backgrounds, so it is natural to expect that the twisted tachyons represent the instability of the orbifold background to decay into flat space. While the instability associated with the bulk tachyon is still mysterious, the bulk tachyon is present both before and after the decay of the cone. The instability we address is the one associated with twisted tachyons. A related situation occurs for the decay of a wrapped D1-brane in bosonic string theory [14. The D1-brane has a spatially constant tachyon mode and additional spatially dependent tachyon modes that signal the instability to decay into a D0-brane. When the D1-brane decays into a D0-brane, the instability associated with the constant mode remains.

The success in the computation of open string tachyon potentials makes it natural to ask if there is a closed string tachyon potential that can be used to understand the decay of orbifold cones. A conjecture concerning the critical points of a twisted tachyon potential was put forward by Dabholkar [15]. The conjecture relates the potential $\mathbb{V}(T)$ for twisted tachyons to the deficit angle $\theta$ of the conical orbifold geometry via the relation $\theta=\kappa^{2} \mathbb{V}(T)$, where $\kappa$ is the gravitational coupling constant. For the $\mathbb{C} / \mathbb{Z}_{N}$ orbifold cone the deficit angle is $\theta_{N}=2 \pi\left(1-\frac{1}{N}\right)$. In the string field theory formulated around the orbifold background, the background itself is represented by tachyons with zero expectation values and the tachyon potential $\mathbb{V}_{N}(T)$ satisfies $\mathbb{V}_{N}(0)=0$. If the endpoint of tachyon condensation is flat space, the final deficit angle is zero. If this background arises for tachyon expectation values $T_{1}$, then $T_{1}$ must be a critical point of the potential that satisfies

$$
\frac{\kappa^{2} \mathbb{V}_{N}\left(T_{1}\right)}{2 \pi\left(1-\frac{1}{N}\right)}=-1
$$

This is the main prediction to be tested. There are similar predictions for critical points that represent transitions to orbifolds with smaller deficit angles. Unless noted otherwise, when we speak of the conjecture, we refer to the expected relation between deficit angles and critical points.

The above conjecture, however, needs refinement. It turns out that expectation values for twisted fields create nonvanishing one-point functions for bulk excitations localized near the apex of the cone. As a result, the physically relevant potential depends on twisted degrees of freedom $\{T\}$ and localized degrees of freedom $\left\{U_{\text {loc }}\right\}$ from the untwisted sector:

$$
\mathbb{V}=\mathbb{V}\left(\{T\} ;\left\{U_{\text {loc }}\right\}\right)
$$

As opposed to conventional potentials $V(\phi)$ that are metric independent and appear in the Lagrangian as $\sqrt{-g} V(\phi)$, the potential $\mathbb{V}$ appears to have metric dependence. This dependence arises because the expectation values for the localized bulk modes are spatially dependent. In addition, even the couplings of twisted fields to the metric do not appear to be conventional since they contain metric 
dependent form factors. The simple argument that relates the potential of twisted fields to the deficit angles (see 2.1) is not strictly valid for a potential $\mathbb{V}$ with metric dependence, so the conjecture (1.1) may not be strictly true. ${ }^{1}$

Despite these caveats, we can frame our present analysis in terms of the conjecture (1.1). In this paper, we truncate the string field to tachyons whose levels are less than or equal to $1 / 4$, a level far below the level of massless fields (level 2). Since massless fields are not included, the possible effects of metric dependence do not feature in our present computations.

We were motivated to compute twisted tachyon potentials in closed string field theory because, to date, there is no evidence that critical points exist, much less, that they have depths proportional to the appropriate change of deficit angle. The issue has been investigated in Type II superstrings for the transition $\mathbb{C} / \mathbb{Z}_{N} \rightarrow \mathbb{C} / \mathbb{Z}_{N-2}$ in the limit of large $N$. Here, according to the conjecture, the tachyon potential should have a critical point at a depth of order $1 / N^{2}$. It has not yet been possible, however, to confirm this expectation [16], although the question remains under active investigation [17, 18]. While these computations are done by naive extrapolation from on-shell CFT results, this may not be a problem since the tachyon which is assumed to be responsible for the decay is nearly massless.

In general, however, the off-shell properties of string field theory are crucial to obtain quantitative results. In fact, to first approximation, the truncation of the open string field to the tachyon gave about $68 \%$ of the D-brane energy [19], a rather encouraging result. Had one used instead the on-shell tachyon action to compute the D-brane energy, the answer would have differed by a factor of $\mathcal{R}^{6}$, where $\mathcal{R}=3 \sqrt{3} / 4$ is a fundamental constant in string field theory [20]. The on-shell action would have predicted about $329 \%$ of the D-brane energy, a rather discouraging result. We will see that even larger off-shell factors arise in closed string field theory. Indeed, while CSFT calculations give about $72 \%$ of the expected depth for the $\mathbb{C} / \mathbb{Z}_{2}$ orbifold, the naive on-shell extrapolation would predict about $1241 \%$ of the expected depth.

Twisted tachyons are expected to be simpler to study than the bulk tachyon, for which no translationally invariant vacuum has been found [20, 21]. Twisted tachyons thus provide a new opportunity to test the calculability of closed string field theory. The viability of the CSFT level expansion is supported by the remarkable fact that cubic interactions of low level give results that are consistent with the deficit angle predictions. We find this encouraging since all successful level-expansion computations have followed this pattern: the key qualitative features and the rough quantitative ones both emerge at low level. We have also examined some quartic interactions, and it appears that they do not destabilize the results obtained with the cubic interactions. We emphasize that it is not yet clear what are the precise rules of level expansion in closed string field theory. It is in fact possible that interactions higher than cubic must be assigned an intrinsic level.

In this paper we have worked with the $\mathbb{C} / \mathbb{Z}_{2}$ orbifold, which can only decay to flat space, and with the $\mathbb{C} / \mathbb{Z}_{3}$ orbifold, which can decay to flat space and to the $\mathbb{C} / \mathbb{Z}_{2}$ orbifold. In both cases, the coupling of the twisted tachyons to localized excitations of the bulk tachyon induce stabilizing quartic terms

\footnotetext{
${ }^{1}$ We would like to thank M. Headrick for drawing our attention to the metric dependence of the potential and for useful discussions on its relevance to the conjecture.
} 
that are needed to create critical points. A rather rough analysis looking for the flat space vacuum gives critical points at about $96 \%$ of the expected depth for $\mathbb{C} / \mathbb{Z}_{2}$ and about $82 \%$ of the expected depth for $\mathbb{C} / \mathbb{Z}_{3}$. With a more detailed analysis that includes bulk tachyon self-interactions, we find about $72 \%$ of the expected depth for $\mathbb{C} / \mathbb{Z}_{2}$ and about $99 \%$ of the expected depth for $\mathbb{C} / \mathbb{Z}_{3}$. We also find the $\mathbb{C} / \mathbb{Z}_{2}$ vacuum on the $\mathbb{C} / \mathbb{Z}_{3}$ tachyon potential at about $134 \%$ of the expected depth. These results are very encouraging but subject to some uncertainly. As we discuss in some detail, it seems possible to increase somewhat the level of the bulk tachyon modes without having to include new twisted fields and higher interactions. If this is done, the critical points become shallower and the predictions are satisfied less accurately. We expect that once new twisted fields and higher interactions are included the critical points will move downwards again. The level expansion will not give a monotonic approach to the expected results. This is a phenomenon familiar from low-level lump computations [14] and from high-level computations of the open string tachyon vacuum 22, 23. It is therefore important to perform higher-level computations in the orbifold backgrounds.

Technically, we need two nontrivial pieces of information. First, we need the relation between the closed string field theory coupling constant and Newton's constant. Second, we need the precisely normalized values for the orbifold correlation functions in the infinite volume limit. ${ }^{2}$ Since our work depends crucially on using the correct numbers, we give the detailed derivations of these results.

\section{The conjecture and closed string field theory}

In the first part of this section we review the conjecture put forward by Dabholkar [15] and discuss the refinements that seem necessary once the unusual properties of the tachyon potential become evident. After a discussion of these matters, we briefly review closed string field theory and state the precise relation between the string field coupling constant and Newton's constant.

\subsection{The conjecture and its refinement}

The conjecture by Dabholkar [15] is motivated by the following effective field theory description of closed strings in an orbifold background:

$$
S=\frac{1}{2 \kappa^{2}} \int d^{D} x \sqrt{-g} e^{-2 \Phi} R-\int_{\mathcal{A}} d^{D-2} x \sqrt{-g^{(D-2)}} e^{-2 \Phi} \mathbb{V}(T) .
$$

Here $\mathbb{V}(T)$ denotes the potential for the twisted tachyon(s) $T, \mathcal{A}$ is the $(D-2)$ dimensional apex of the cone, and $g^{(D-2)}$ is the determinant of the metric induced on $\mathcal{A}$ by the full spacetime metric. The twisted sector fields live on $\mathcal{A}$.

We look for backgrounds where the dilaton $\Phi$ is constant over spacetime, the metric describes the conical geometry $\mathbb{R}^{D-2} \times\left(\mathbb{C} / \mathbb{Z}_{N}\right)$, and the twisted tachyons are constant over $\mathcal{A}$. If the dilaton is constant, its field equation requires

$$
\sqrt{-g} R=\left(2 \kappa^{2}\right) \mathbb{V}(T) \sqrt{-g^{(D-2)}} \delta^{(2)}(x) .
$$

\footnotetext{
${ }^{2}$ Some of the CFT computations in $\$$ have also been performed independently in 17 .
} 
For the conical geometry the metric factorizes into a flat $(D-2)$ metric $g_{\alpha \beta}=\eta_{\alpha \beta}$ and a nontrivial twodimensional metric $g_{i j}$. For this spacetime metric $R_{\alpha \beta}=0$, and $R$ is equal to the scalar curvature of the two-dimensional cone: $R=R^{(2)}=g^{i j} R_{i j}$. Moreover $g=g^{(D-2)} g^{(2)}$, where $g^{(2)}$ is the determinant of $g_{i j}$. The above equation thus becomes

$$
\sqrt{g^{(2)}} R^{(2)}=\left(2 \kappa^{2}\right) \mathbb{V}(T) \delta^{(2)}(x) .
$$

Since the tachyons $T$ are constants on $\mathcal{A}$, the above right-hand side is constant over $\mathcal{A}$, as required by consistency given that the left-hand side is also constant over $\mathcal{A}$. For a cone of deficit angle $\theta$ (or total angle $2 \pi-\theta$ ), one has $\sqrt{g^{(2)}} R^{(2)}=2 \theta \delta^{(2)}(x)$, so (2.3) is satisfied when the deficit angle and the potential are related by

$$
\theta=\kappa^{2} \mathbb{V}(T)
$$

The gravitational field equations are also satisfied by the conical background geometry. The nontrivial components of Einstein's equations are

$$
\sqrt{-g} R_{\alpha \beta}-\frac{1}{2} g_{\alpha \beta}\left(\sqrt{-g} R-2 \kappa^{2} \mathbb{V}(T) \sqrt{-g^{(D-2)}} \delta^{(2)}(x)\right)=0, \quad R_{i j}-\frac{1}{2} g_{i j} R=0 .
$$

The dilaton equation (2.2) implies that the object inside parentheses in the first equation vanishes. As a result, we find $R_{\alpha \beta}=0$, which is satisfied by our metric. With vanishing $R_{\alpha \beta}$, the second equation is identically satisfied for a two-dimensional cone.

For a transition between an initial configuration with deficit angle $\theta_{i}$ and tachyon values $T_{i}$ and a final configuration with deficit angle $\theta_{f}$ and tachyon values $T_{f}$, equation (2.4) gives

$$
\frac{\kappa^{2}\left(\mathbb{V}\left(T_{i}\right)-\mathbb{V}\left(T_{f}\right)\right)}{\theta_{i}-\theta_{f}}=1 .
$$

The deficit angle $\theta_{N}$ for the $\mathbb{C} / \mathbb{Z}_{N}$ orbifold cone is $\theta_{N}=2 \pi\left(1-\frac{1}{N}\right)$. Assume we begin with this orbifold background, represented by tachyons with expectation values $T_{N}$. If the endpoint of tachyon condensation is flat space, the final deficit angle is zero. Let $T_{1}$ denote the expectation values of the tachyons at a critical point that represents flat space. Equation (2.6) then predicts that the following dimensionless ratio must be equal to minus one:

$$
\frac{\kappa^{2}\left(\mathbb{V}\left(T_{1}\right)-\mathbb{V}\left(T_{N}\right)\right)}{2 \pi\left(1-\frac{1}{N}\right)}=-1 .
$$

For a transition from the $\mathbb{C} / \mathbb{Z}_{N}$ orbifold to the $\mathbb{C} / \mathbb{Z}_{M}$ orbifold, with $2 \leq M<N$, equation (2.6) predicts that

$$
\frac{\kappa^{2}\left(\mathbb{V}\left(T_{M}\right)-\mathbb{V}\left(T_{N}\right)\right)}{2 \pi\left(\frac{1}{M}-\frac{1}{N}\right)}=-1 .
$$

Here $T_{M}$ denotes the expectation values of the tachyons at the critical point that represents the $\mathbb{C} / \mathbb{Z}_{M}$ orbifold. For any fixed $\mathbb{C} / \mathbb{Z}_{N}$ orbifold, the above ratios provide $N-1$ tests of the calculated tachyon potential. When we formulate the string field theory of the $\mathbb{C} / \mathbb{Z}_{N}$ orbifold, the tachyon expectation 
values $T_{N}$ that represent the orbifold itself are all zero. The tachyon potential, denoted henceforth as $\mathbb{V}_{N}(T)$, automatically satisfies $\mathbb{V}_{N}\left(T_{N}\right)=0$. It is therefore useful to define a normalized potential by

$$
f_{N}(T) \equiv \frac{\kappa^{2} \mathbb{V}_{N}(T)}{2 \pi\left(1-\frac{1}{N}\right)}
$$

so that the predictions in (2.7) and (2.8) can be summarized as

$$
f_{N}\left(T_{M}\right)=-\frac{\left(\frac{1}{M}-\frac{1}{N}\right)}{\left(1-\frac{1}{N}\right)}, \quad M=1,2, \ldots, N-1 .
$$

We will study in detail the $\mathbb{C} / \mathbb{Z}_{2}$ orbifold, for which we have one test:

$$
f_{2}(T) \equiv \frac{1}{\pi} \kappa^{2} \mathbb{V}_{2}(T), \quad f_{2}\left(T_{1}\right) \stackrel{?}{=}-1 .
$$

For the $\mathbb{C} / \mathbb{Z}_{3}$ orbifold, which we also examine in detail, we have two tests:

$$
f_{3}(T) \equiv \frac{3}{4 \pi} \kappa^{2} \mathbb{V}_{3}(T), \quad f_{3}\left(T_{1}\right) \stackrel{?}{=}-1, \quad f_{3}\left(T_{2}\right) \stackrel{?}{=}-\frac{1}{4} .
$$

For any $\mathbb{C} / \mathbb{Z}_{N}$ orbifold the values $f_{N}\left(T_{1}\right), f_{N}\left(T_{2}\right), \ldots, f_{N}\left(T_{N-1}\right)$ give the depths of the various critical points in the tachyon potential, normalized using the expected depth of the point that represents the transition to flat space.

The relation (2.4) between the deficit angle and the potential $\mathbb{V}$ is in fact familiar in other guises. Indeed, for a cosmic string in four-dimensional spacetime one has $\theta=8 \pi G \mu$, where $8 \pi G=\kappa^{2}$ and $\mu$ is the energy per unit length of the string [24, 25]. In three-dimensional gravity, one has $\theta=8 \pi G M$, where $M$ is the mass of a point particle that creates a conical geometry with deficit angle $\theta$ [26]. The energy associated with $\mathbb{V}$, or its lower dimensional versions for strings or point particles, may have an intrinsic gravitational meaning. It has been demonstrated that spaces whose asymptotic geometry is conical can be assigned an energy through a comparison with asymptotically flat space [27]. This energy agrees with value of the potential $\mathbb{V}$.

The explicit analysis in the following sections demonstrates that the above conjecture needs refinement. The relevant potential is not just a potential for all the scalars in the twisted sector. Expectation values for twisted fields create nonvanishing one-point functions for modes of bulk fields that live near the apex of the cone. It follows that these modes must be excited and, as we shall see, they contribute to the potential. The bulk tachyon, for example, acquires some expectation value - roughly a Gaussian that decays quickly away from the apex of the cone. We claim that the physically relevant potential depends on twisted fields and localized degrees of freedom from the untwisted sector:

$$
\mathbb{V}=\mathbb{V}\left(\{T\} ;\left\{U_{\text {loc }}\right\}\right)
$$

where $\{T\}$ denotes the infinite collection of twisted fields (thus the $T$ ), and $\left\{U_{\text {loc }}\right\}$ denotes the infinite collection of localized excitations in the untwisted sector (thus the $U$ ). At least for bosonic strings, the existence of a critical point seems to require both kinds of excitations. 
In general, the change of deficit angle in the process of tachyon condensation cannot be read directly from a potential of the type indicated in (2.13). To explain this consider a scalar $\phi$ minimally coupled to pure gravity, with a scalar potential $V(\phi)$ that, as usual, is metric independent. The problem of finding a vacuum is easily solved in two steps. One finds the critical points of the potential $V(\phi)$ and sets $\phi$ equal to any of the critical values. The potential evaluated at the chosen critical point defines the cosmological term. The gravitational equations can then be solved to find the appropriate de Sitter or anti-de Sitter background. Similar remarks apply to the idealized system described by the action (2.1): the values of the potential are directly correlated to the observable deficit angle of the cone. The problem arises for the potential (2.13) because the contributions from the localized bulk fields depend nontrivially on the spacetime metric; the corresponding expectation values are obtained by solving equations of motion with metric dependent kinetic terms. The (unusual) metric dependence of the potential $\mathbb{V}$ implies that its minimization cannot be disentangled from the problem of solving the gravitational equations. Even if we calculated $\mathbb{V}$ to full accuracy using the fixed metric of the original orbifold, the critical values of $\mathbb{V}$ need not reproduce the precise expectations for the deficit angles. Indeed, at any critical point where a new orbifold would be expected, the metric is different from the original one, and the potential is not strictly applicable.

In computations of $\mathbb{V}$ that only include fields with levels lower than two, one is in effect using the background metric of the original orbifold. Since the massless closed string fields appear at level two, neither metric changes nor gravitational back-reaction feature in the low-level calculations that we perform in the present paper. The issue of metric dependence does not arise and one is then justified in relating the depth of critical points in $\mathbb{V}$ to changes in deficit angles. This is what we do in the sections that follow.

\subsection{Closed string field theory and Newton's constant}

In this section we briefly recall the structure of the closed string field theory action and give the computational rules for the quadratic and cubic terms. Some discussion of the quartic couplings will be given in 86 . While most of our results will be general, the theory around flat space will receive particular attention. The formulation of CSFT for the orbifold background will be discussed in the later sections.

The closed string field theory action around flat space is given in [10]:

$$
S=-\frac{2}{\alpha^{\prime}}\left(\frac{1}{2}\left\langle\Psi, c_{0}^{-} Q \Psi\right\rangle+\frac{1}{3 !} \kappa\langle\Psi, \Psi, \Psi\rangle+\frac{1}{4 !} \kappa^{2}\langle\Psi, \Psi, \Psi, \Psi\rangle+\ldots\right),
$$

where the dots represent additional interactions - the theory is fully nonpolynomial. The closed string field $|\Psi\rangle$ is a ghost number two vector of the CFT state space and satisfies the constraints $\left(b_{0}-\bar{b}_{0}\right)|\Psi\rangle=$ $\left(L_{0}-\bar{L}_{0}\right)|\Psi\rangle=0$. The above action, as opposed to that in [10], includes the explicit $\alpha^{\prime}$ dependence. We use a spacetime metric with signature $(-,+,+, \ldots,+)$ and $c_{0}^{ \pm}=\frac{1}{2}\left(c_{0} \pm \bar{c}_{0}\right)$. The BRST operator $Q$ is normalized by writing $Q=c_{0} L_{0}+\bar{c}_{0} \bar{L}_{0}+\ldots=c_{0}^{+}\left(L_{0}+\bar{L}_{0}\right)+c_{0}^{-}\left(L_{0}-\bar{L}_{0}\right)+\ldots$, where the dots indicate terms that involve neither $c_{0}$ nor $\bar{c}_{0}$. For tachyon states $c_{1} \bar{c}_{1}|p\rangle$ of momentum $p$ we have $L_{0}=\bar{L}_{0}=-1+\frac{1}{4} \alpha^{\prime} p^{2}$. The momentum operator $\widehat{p}$ is Hermitian: $\widehat{p}^{\dagger}=\widehat{p}$, but under BPZ 
conjugation $\widehat{p} \rightarrow-\widehat{p}$. This means that Hermitian conjugation takes $|p\rangle \rightarrow\langle p|$ while BPZ conjugation takes $|p\rangle \rightarrow\langle-p|$. The BRST operator is Hermitian, and the above action is real if the Hermitian conjugate of the string field $\Psi$ is equal to minus the BPZ conjugate of the field.

We define the inner product $\langle\cdot, \cdot\rangle$ in terms of BPZ conjugation and an overlap between bras and kets: $\langle A, B\rangle=\langle b p z(A) \mid B\rangle$. In the theory formulated around flat spacetime, the basic overlap is

$$
\left\langle p^{\prime}\left|c_{-1} \bar{c}_{-1} c_{0}^{-} c_{0}^{+} c_{1} \bar{c}_{1}\right| p\right\rangle=(2 \pi)^{D} \delta^{(D)}\left(p-p^{\prime}\right) .
$$

With ghost fields $c(z)=\sum_{n} c_{n} z^{-n+1}$ and $\bar{c}(\bar{z})=\sum_{n} \bar{c}_{n} \bar{z}^{-n+1}$ one has

$$
\left\langle p^{\prime}\left|c\left(z_{1}\right) \bar{c}\left(\bar{z}_{1}\right) c\left(z_{2}\right) \bar{c}\left(\bar{z}_{2}\right) c\left(z_{3}\right) \bar{c}\left(\bar{z}_{3}\right)\right| p\right\rangle=2(2 \pi)^{D} \delta^{(D)}\left(p-p^{\prime}\right)\left|z_{1}-z_{2}\right|^{2}\left|z_{1}-z_{3}\right|^{2}\left|z_{2}-z_{3}\right|^{2} .
$$

For the purposes of the present paper it suffices to consider string fields of the form $|\Psi\rangle=$ $\sum_{\alpha} c_{1} \bar{c}_{1} \phi^{\alpha}\left|\mathcal{O}_{\alpha}\right\rangle$, where $\alpha$ is an index that can take both continuous and discrete values, $\phi^{\alpha}$ is a component field, and $\left|\mathcal{O}_{\alpha}\right\rangle$ is a primary state of the matter conformal field theory with conformal dimensions $h_{\alpha}=\bar{h}_{\alpha}$. For this string field, the quadratic and cubic terms in the action are

$$
S=-\frac{2}{\alpha^{\prime}} \sum_{\alpha, \beta}\left(h_{\beta}-1\right) \phi^{\alpha} m_{\alpha \beta} \phi^{\beta}-\frac{1}{3 !} \frac{2 \kappa}{\alpha^{\prime}} \sum_{\alpha, \beta, \gamma} \mathcal{R}^{6-2\left(h_{\alpha}+h_{\beta}+h_{\gamma}\right)} \phi^{\alpha} \phi^{\beta} \phi^{\gamma} \mathcal{C}_{\alpha \beta \gamma} .
$$

Here $m_{\alpha \beta}=\left\langle b p z\left(\mathcal{O}_{\alpha}\right)\left|c_{-1} \bar{c}_{-1} c_{0}^{-} c_{0}^{+} c_{1} \bar{c}_{1}\right| \mathcal{O}_{\beta}\right\rangle$ and the constant $\mathcal{C}_{\alpha \beta \gamma}$ is defined by the CFT correlator

$$
\left\langle c \bar{c} \mathcal{O}_{\alpha}(0) c \bar{c} \mathcal{O}_{\beta}(1) c \bar{c} \mathcal{O}_{\gamma}(\infty)\right\rangle=\mathcal{C}_{\alpha \beta \gamma} .
$$

This correlator is evaluated in the complex plane $z$, with local coordinates $z, z-1$, and $1 / z$ around the points 0,1 , and $\infty$, respectively. The prescription (2.17) for the evaluation of the cubic term follows from the geometrical description of the three-closed-string vertex. The vertex can be viewed as the Riemann sphere $\widehat{\mathbb{C}}$ punctured at $1 / \sqrt{3}, \omega / \sqrt{3}$, and $\omega^{2} / \sqrt{3}$, where $\omega=\exp (2 \pi i / 3)$. The distance between any two punctures is one. The radial lines departing $z=0$ at angles $\pm \pi / 3$ and $-\pi$ cut the sphere into three punctured disks. The constant $\mathcal{R}$ is the inverse of the mapping radius ${ }^{3} \rho$ of any of these three punctured disks:

$$
\mathcal{R} \equiv \frac{1}{\rho}=\frac{3 \sqrt{3}}{4} \simeq 1.2990
$$

The constant $\mathcal{R}$ plays an important role in any off-shell amplitude. Indeed, the $\mathcal{R}$ factor in the cubic term is the product of factors of the form $\mathcal{R}^{2(1-h)}$, one for each operator $c_{1} \bar{c}_{1} \mathcal{O}$. For an on-shell operator, the factor becomes unity since $h=1$. For off-shell operators, however, the factors of $\mathcal{R}$ can have a significant effect.

Consider now the CSFT action in $D=26$, evaluated for the tachyon string field:

$$
|\Psi\rangle=\int \frac{d^{D} p}{(2 \pi)^{D}} u(p) c_{1} \bar{c}_{1}|p\rangle, \quad u(p)=\int d^{D} x u(x) e^{-i p \cdot x}, \quad u^{*}(p)=u(-p) .
$$

\footnotetext{
${ }^{3}$ The mapping radius $\rho$ of a disk $D$ with coordinate $z$ at the puncture is given by $|d z / d \xi|$, evaluated at the puncture, where $z(\xi)$ is the conformal map from a unit disk $|\xi|=1$ punctured at the origin to $D$. See [20] for more details.
} 
Using the rules described above, the quadratic and cubic terms in the action take the form

$$
-\frac{1}{2} \int \frac{d^{D} p}{(2 \pi)^{D}} u(-p)\left(p^{2}-\frac{4}{\alpha^{\prime}}\right) u(p)-\frac{1}{3 !} \frac{4 \kappa}{\alpha^{\prime}} \int \prod_{i=1}^{3}\left[\frac{d^{D} p_{i}}{(2 \pi)^{D}} \mathcal{R}^{2-\frac{1}{2} \alpha^{\prime} p_{i}^{2}} u\left(p_{i}\right)\right](2 \pi)^{D} \delta^{(D)}\left(p_{1}+p_{2}+p_{3}\right) .
$$

In coordinate space this tachyon action $S_{u}$ becomes

$$
S_{u}=\int d^{D} x\left\{-\frac{1}{2} \eta^{\mu \nu} \partial_{\mu} u \partial_{\nu} u-\frac{1}{2}\left(-\frac{4}{\alpha^{\prime}}\right) u^{2}-\frac{1}{3 !} \frac{4 \kappa}{\alpha^{\prime}}\left[\mathcal{R}^{2+\frac{1}{2} \alpha^{\prime} \partial^{2}} u\right]^{3}\right\} .
$$

The S-matrix element for the scattering of three on-shell tachyons is therefore

$$
\mathcal{S}=-i \frac{4 \kappa}{\alpha^{\prime}}(2 \pi)^{D} \delta^{(D)}\left(\sum_{i=1}^{3} p_{i}\right)
$$

This result can be compared with the familiar CFT calculation for this amplitude in terms of the conventional gravitational coupling constant (see [28, equations (6.6.9) and (6.6.18), and note the difference in overall sign for the tachyon field). One finds that the coupling $\kappa$ in the CSFT action is the gravitational coupling constant. ${ }^{4}$ The spacetime gravitational action that emerges from the closed string field theory is therefore given by the first term in (2.1).

The tachyon potential $\mathbb{V}(u)$ is obtained from $S_{u}$ by taking all spacetime derivatives to vanish:

$$
\mathbb{V}(u)=-\frac{1}{2}\left(\frac{4}{\alpha^{\prime}}\right) u^{2}+\frac{1}{3 !} \frac{4 \kappa}{\alpha^{\prime}} \mathcal{R}^{6} u^{3} .
$$

This potential has a nonvanishing critical point $u_{*}$ and

$$
\mathbb{V}\left(u_{*}\right)=\frac{1}{2 \kappa^{2}} \frac{1}{\alpha^{\prime}}\left(-\frac{16}{3 \mathcal{R}^{12}}\right) \simeq \frac{1}{2 \kappa^{2}} \frac{1}{\alpha^{\prime}}(-0.23096) .
$$

The meaning of this critical point, first found in [29], is not clear. The critical point in fact disappears upon inclusion of the quartic term in the tachyon potential [20, 21]. ${ }^{5}$

In open string field theory (OSFT) level expansion has proven to be a surprisingly good approximation scheme. The level $\ell$ of a state is defined to be equal to the $L_{0}$ eigenvalue of the state plus one. As a result, the zero-momentum open string tachyon has level zero. The level of the spacetime field associated with a state is declared to be equal to the level of the state. For closed strings we define

$$
\ell \equiv L_{0}+\bar{L}_{0}+2
$$

It follows that the level of the primary state $c_{1} \bar{c}_{1}\left|\mathcal{O}_{\alpha}\right\rangle$ is

$$
\ell\left(c_{1} \bar{c}_{1}\left|\mathcal{O}_{\alpha}\right\rangle\right)=2 h_{\alpha}
$$

\footnotetext{
${ }^{4}$ In four spacetime dimensions $\kappa^{2}=8 \pi G$, where $G$ is Newton's constant.

${ }^{5}$ It has been suggested by Bergman [30 that $\mathbb{V}\left(u_{*}\right)$ represents a cosmological constant that may be compared with the noncritical string cosmological term $\Lambda=-\frac{1}{2 \kappa^{2}} \frac{1}{\alpha^{\prime}} \frac{2(D-26)}{3}$ (see [28], equation (3.7.20)) for $D \leq 2$. This value of $\Lambda$ for $D=2$, however, is approximately 70 times larger than the value of $\mathbb{V}\left(u_{*}\right)$. This comparison, if valid, probably requires a re-computation of the lower-dimensional gravitational coupling.
} 
For example, the level of the closed string tachyon state $c_{1} \bar{c}_{1}|p\rangle$ with momentum $p$ is

$$
\ell\left(c_{1} \bar{c}_{1}|p\rangle\right)=\frac{1}{2} \alpha^{\prime} p^{2}
$$

In this convention, the level of a massless closed string state is equal to two. The level of a cubic closed string interaction is defined as in OSFT: it is equal to the sum of levels of the operators that appear in the interaction. The level of the operators does in fact enter into the explicit form of the cubic term. For primary fields a cubic interaction of total level $L$ contains a suppression factor $1 / \mathcal{R}^{L}$, as can be seen from (2.17). This suppression is presumably one of the various ingredients that make level expansion work. We suspect that quartic and higher elementary interactions in CSFT carry intrinsic level. This would mean that the total level of any such interaction would be given by the sum of levels of the operators plus the intrinsic level carried by the interaction. The intrinsic level, for example, may be given by a constant times $(k-3)$, where $k$ is the order of the interaction. If this turns out to be the case, finite level computations will only include a finite set of interactions despite the nonpolynomiality of the theory.

\section{Orbifold CFT correlators}

In this section we compute correlators of the orbifold CFT [31, 32, 33] which are necessary for our computations of the tachyon potentials. Much of the needed work can be taken directly from the detailed work of Dixon et al. 31]. Since precise numerical values are crucial in our work, we introduce the necessary factors of $\alpha^{\prime}$ in all of the formulae - this also helps keep track of units. Moreover, we must take the infinite volume limit of the results in 31], which are given for two-dimensional orbifolds with finite volume $V_{\Lambda} \cdot{ }^{6}$ Correlators of three twist fields, for example, are proportional to $\sqrt{V_{\Lambda}}$ and appear to become infinite when $V_{\Lambda} \rightarrow \infty$, but proper attention to the normalization of the operators involved shows that effectively the volume $V_{\Lambda}$ is replaced by a finite constant.

Consider the two dimensional plane spanned by the string coordinates $X^{1}$ and $X^{2}$. This plane can be described as the complex plane $\mathbb{C}$ with a complex coordinate $\mathrm{X}$ :

$$
\mathrm{X}=X^{1}+i X^{2}, \quad \overline{\mathrm{X}}=X^{1}-i X^{2} .
$$

The orbifold $\mathbb{C} / \mathbb{Z}_{N}$ is obtained by $\mathbb{Z}_{N}$ action on the complex coordinate $\mathrm{X}$. The generator of this action takes $\mathrm{X}$ to $e^{2 \pi i / N} \mathrm{X}$. The quotient space is a cone (orbifold) whose total angle at the apex is $2 \pi / N$; the deficit angle is $2 \pi\left(1-\frac{1}{N}\right)$. It is a noncompact orbifold of infinite volume. In the orbifold CFT there are twist fields $\sigma_{k}(z, \bar{z})$ labelled by the integer $k$, with $0<k<N$. If we place $\sigma_{k}$ at $z=0$, the coordinate field has the monodromy $\mathrm{X}\left(e^{2 \pi i} z, e^{-2 \pi i} \bar{z}\right)=e^{2 \pi i k / N} \mathrm{X}(z, \bar{z})$. The conformal dimensions of $\sigma_{k}$ are

$$
h_{k}=\bar{h}_{k}=\frac{1}{2} \frac{k}{N}\left(1-\frac{k}{N}\right) .
$$

\footnotetext{
${ }^{6}$ Some aspects of conformal field theory for noncompact spacetimes were discussed in 34 .
} 
The relevant CFT is the full CFT obtained as the tensor product of the CFT which describes the flat $D-2$ dimensions and the orbifold CFT of the cone (the inclusion of the ghost CFT causes no significant change). The twist fields are normalized so that two-point functions on the sphere are given by

$$
\left\langle\sigma_{N-k}(\infty) \sigma_{k}(0)\right\rangle=V_{D-2} .
$$

The insertion at $z=\infty$, as usual, is defined by an insertion at $w=0$, with $w=1 / z$. When the subscripts of the twist fields do not add up to $N$ the correlator vanishes. The factor $V_{D-2}$ denotes the volume of the flat lower-dimensional spacetime. This factor is necessary because the twist operators can be dressed with momenta $q$ in the $D-2$ directions in which case

$$
\left\langle e^{i q \cdot X} \sigma_{N-k}(\infty) e^{i q^{\prime} \cdot X} \sigma_{k}(0)\right\rangle=(2 \pi)^{D-2} \delta^{(D-2)}\left(q+q^{\prime}\right) .
$$

It is customary to fix some $k$ that satisfies $0<k \leq N / 2$ and to call $\sigma_{+} \equiv \sigma_{k}$. Additionally, we let $\sigma_{-} \equiv \sigma_{N-k}$, which is reasonable since the monodromies around $\sigma_{-}$and $\sigma_{+}$are inverses of each other. The twist fields $\sigma_{+}$and $\sigma_{-}$have identical dimensions, given by (3.2). Finally, one introduces 'doubly' twisted fields: $\sigma_{++} \equiv \sigma_{2 k}$ and $\sigma_{--} \equiv \sigma_{N-2 k}$ for $0<k<N / 2$, which also have identical dimensions. On account of (3.3), $\left\langle\sigma_{-}(\infty) \sigma_{+}(0)\right\rangle=\left\langle\sigma_{--}(\infty) \sigma_{++}(0)\right\rangle=V_{D-2}$.

The orbifold CFT admits 'untwisted' operators that carry momentum along the directions on the cone. These operators are needed to represent bulk fields that live everywhere, including the apex of the cone. Using the momenta $p_{1}$ and $p_{2}$ associated with $X^{1}$ and $X^{2}$, respectively, we introduce the complex combinations:

$$
\mathrm{p}=\frac{1}{2}\left(p_{1}-i p_{2}\right), \quad \overline{\mathrm{p}}=\frac{1}{2}\left(p_{1}+i p_{2}\right) \quad \rightarrow \quad p_{1} X^{1}+p_{2} X^{2}=\mathrm{pX}+\overline{\mathrm{p}} \overline{\mathrm{X}} .
$$

We also define $p^{2} \equiv p_{1}^{2}+p_{2}^{2}=4 \overline{\mathrm{p}} \mathrm{p}$. The vertex operator

$$
\mathcal{U}_{\mathrm{p}} \equiv e^{i \mathrm{p} \mathrm{X}+i \overline{\mathrm{p}} \overline{\mathrm{X}}}
$$

does not belong to the orbifold CFT since it is not invariant under $\mathrm{p} \rightarrow \alpha \mathrm{p}$ with $\alpha=\exp (2 \pi i / N)$. An invariant vertex operator $\mathcal{V}_{\mathrm{p}}$ is readily defined:

$$
\mathcal{V}_{\mathrm{p}} \equiv \frac{1}{N} \sum_{i=0}^{N-1} \mathcal{U}_{\alpha^{i} \mathrm{p}}=\frac{1}{N}\left(\mathcal{U}_{\mathrm{p}}+\mathcal{U}_{\alpha \mathrm{p}}+\cdots+\mathcal{U}_{\alpha^{N-1} \mathrm{p}}\right) \quad \rightarrow \quad \mathcal{V}_{\alpha \mathrm{p}}=\mathcal{V}_{\mathrm{p}}
$$

A complete set of independent operators $\mathcal{V}_{\mathrm{p}}$ can be chosen by restricting $\mathrm{p}$ to any fundamental domain $\Gamma$ of the identification $\mathrm{p} \sim \alpha \mathrm{p}$. The operators $\mathcal{U}_{\mathrm{p}}$ (and $\mathcal{V}_{\mathrm{p}}$ ) have dimensions $h=\bar{h}=\frac{1}{4} \alpha^{\prime} p^{2}=\alpha^{\prime} \overline{\mathrm{p}} \mathrm{p}$ and are normalized such that

$$
\left\langle\mathcal{U}_{\mathrm{p}}(\infty) \mathcal{U}_{\mathrm{p}^{\prime}}(0)\right\rangle=(2 \pi)^{2} \delta^{(2)}\left(p+p^{\prime}\right) V_{D-2} \equiv(2 \pi)^{2} \delta\left(p_{1}+p_{1}^{\prime}\right) \delta\left(p_{2}+p_{2}^{\prime}\right) V_{D-2} .
$$

This equation must be viewed as a computational device to obtain two-point functions of well-defined operators of the orbifold CFT. Since $\mathcal{U}_{0}=1$, we find

$$
\left\langle\mathcal{U}_{\mathrm{p}}(0)\right\rangle=(2 \pi)^{2} \delta^{(2)}(p) V_{D-2} .
$$


We can use the above discussion to produce the vertex operator for a general configuration of the bulk tachyon. This is given by a superposition of states of various momenta $q, p_{1}$, and $p_{2}$ :

$$
\int \frac{d^{D-2} q}{(2 \pi)^{D-2}} \int_{\Gamma} \frac{d^{2} p}{(2 \pi)^{2}} N u(q, \mathrm{p}) c \bar{c} e^{i q \cdot X} \mathcal{V}_{\mathrm{p}}
$$

where we included a factor of $N$ and integrated over the fundamental domain $\Gamma$, with $d^{2} p \equiv d p_{1} d p_{2}$. The weight function $u(q, \mathrm{p})$ will be eventually identified as the bulk tachyon field. Using the definition of $\mathcal{V}_{\mathrm{p}}$ we find

$$
\int \frac{d^{D-2} q}{(2 \pi)^{D-2}} \int_{\Gamma} \frac{d^{2} p}{(2 \pi)^{2}} u(q, \mathrm{p}) c \bar{c} e^{i q \cdot X}\left(\mathcal{U}_{\mathrm{p}}+\mathcal{U}_{\alpha \mathrm{p}}+\cdots+\mathcal{U}_{\alpha^{N-1} \mathrm{p}}\right)
$$

It is convenient to extend the definition of the function $u(q, \mathrm{p})$ by setting $u(q, \alpha \mathrm{p})=u(q$, $\mathrm{p})$. This definition allows us to rewrite the vertex operator as

$$
\text { bulk-tachyon vertex operator: } \quad \int \frac{d^{D-2} q}{(2 \pi)^{D-2}} \int \frac{d^{2} p}{(2 \pi)^{2}} u(q, p) c \bar{c} e^{i q \cdot X} \mathcal{U}_{\mathrm{p}} \text {. }
$$

Here the two-dimensional integration is unrestricted and, for simplicity of notation, we write $u(q, p)$ instead of $u(q, \mathrm{p})$. This vertex operator will be of utility in 4 and 95 .

Let us now discuss operator product coefficients and three-point functions that involve twist fields. We introduce coefficients $C_{-,+}^{\mathcal{V}}(\mathrm{p})$, with $\mathrm{p} \in \Gamma$, using the operator product expansion (OPE) of $\sigma_{-}$ and $\sigma_{+}$:

$$
\sigma_{-}(x, \bar{x}) \sigma_{+}(0) \sim \int_{\Gamma} \frac{d^{2} p}{(2 \pi)^{2}} N C_{-,+}^{\mathcal{V}}(\mathrm{p})|x|^{2 \alpha^{\prime} \overline{\mathrm{p}}-\frac{2 k}{N}\left(1-\frac{k}{N}\right)} \mathcal{V}_{\mathrm{p}}(0)+\ldots,
$$

where the dots denote terms with operators that have vanishing one-point functions and vanishing two-point functions with $\mathcal{V}_{\mathrm{p}}$. If we define $C_{-,+}^{\mathcal{V}}(\mathrm{p})$ for any p by requiring $C_{-,+}^{\mathcal{V}}(\alpha \mathrm{p})=C_{-,+}^{\mathcal{V}}(\mathrm{p})$, then (3.13) can be rewritten as follows:

$$
\sigma_{-}(x, \bar{x}) \sigma_{+}(0) \sim \int \frac{d^{2} p}{(2 \pi)^{2}} C_{-,+}^{\mathcal{V}}(\mathrm{p})|x|^{2 \alpha^{\prime} \overline{\mathrm{p}}-\frac{2 k}{N}\left(1-\frac{k}{N}\right)} \mathcal{U}_{\mathrm{p}}(0)+\ldots
$$

It follows from this OPE that

$$
\left\langle\sigma_{-}(1) \sigma_{+}(0)\right\rangle=\int \frac{d^{2} p}{(2 \pi)^{2}} C_{-,+}^{\mathcal{V}}(\mathrm{p})\left\langle\mathcal{U}_{\mathrm{p}}(0)\right\rangle=C_{-,+}^{\mathcal{V}}(0) V_{D-2} .
$$

Therefore,

$$
C_{-,+}^{\mathcal{V}}(0)=1
$$

Let us define the three-point function $C_{\mathcal{V},-,+}(\mathrm{p})$ by

$$
C_{\mathcal{V},-,+}(\mathrm{p})=\left\langle\mathcal{V}_{\mathrm{p}}(\infty) \sigma_{-}(1) \sigma_{+}(0)\right\rangle
$$


We can relate $C_{\mathcal{V},-,+}(\mathrm{p})$ to the OPE coefficient $C_{-,+}^{\mathcal{V}}(-\mathrm{p})$ using (3.14) with $x=1$ :

$$
\begin{aligned}
C_{\mathcal{V},-,+}(\mathrm{p}) & =\int \frac{d^{2} p^{\prime}}{(2 \pi)^{2}} C_{-,+}^{\mathcal{V}}\left(\mathrm{p}^{\prime}\right)\left\langle\mathcal{V}_{\mathrm{p}}(\infty) \mathcal{U}_{\mathrm{p}^{\prime}}(0)\right\rangle \\
& =\frac{1}{N} \sum_{i=0}^{N-1} \int \frac{d^{2} p^{\prime}}{(2 \pi)^{2}} C_{-,+}^{\mathcal{V}}\left(\mathrm{p}^{\prime}\right)\left\langle\mathcal{U}_{\alpha^{i} \mathrm{p}}(\infty) \mathcal{U}_{\mathrm{p}^{\prime}}(0)\right\rangle \\
& =\frac{1}{N} \sum_{i=0}^{N-1} C_{-,+}^{\mathcal{V}}\left(-\alpha^{i} \mathrm{p}\right) V_{D-2}=C_{-,+}^{\mathcal{V}}(-\mathrm{p}) V_{D-2}
\end{aligned}
$$

The three-point function $C_{\mathcal{V},-,+}(\mathrm{p})$ is effectively the one-point function of an operator of the form $\exp (i \mathrm{pX}+i \overline{\mathrm{p}} \overline{\mathrm{X}})$, with twisted boundary conditions. It is given by an exponential of the propagator $-\overline{\mathrm{p}} \mathrm{p}\langle\overline{\mathrm{X}} \mathrm{X}\rangle$ evaluated with twisted boundary conditions, so it has a Gaussian dependence on the momentum. Since the overall normalization of $C_{\mathcal{V},-,+}(\mathrm{p})$ is fixed by (3.16) and (3.18), we can write

$$
C_{\mathcal{V},-,+}(\mathrm{p})=V_{D-2} \delta^{\prime-\alpha^{\prime} \overline{\mathrm{p}}}, \quad C_{-,+}^{\mathcal{V}}(\mathrm{p})=\delta^{\prime-\alpha^{\prime} \overline{\mathrm{p}}}
$$

where $\delta^{\prime}$ is a constant to be determined.

For the two-dimensional orbifold of infinite volume, the correlator of four twist operators is given in [31:

$$
Z(x, \bar{x})=\left\langle\sigma_{-}(\infty) \sigma_{+}(1) \sigma_{-}(x, \bar{x}) \sigma_{+}(0)\right\rangle=\frac{\mathcal{N}|x(1-x)|^{-\frac{2 k}{N}\left(1-\frac{k}{N}\right)}}{F(x) F(1-\bar{x})+F(1-x) F(\bar{x})},
$$

where $F(x) \equiv F\left(\frac{k}{N}, 1-\frac{k}{N} ; 1 ; x\right)$ is a hypergeometric function. To determine the normalization constant $\mathcal{N}$ we examine the behavior of $Z(x, \bar{x})$ when $x \sim 0$. On the one hand, using the OPE (3.13) and (3.19) we find

$$
\begin{aligned}
Z(x, \bar{x}) & \sim \int_{\Gamma} \frac{d^{2} p}{(2 \pi)^{2}} N C_{-,+}^{\mathcal{V}}(\mathrm{p})|x|^{2 \alpha^{\prime} \overline{\mathrm{p}}-\frac{2 k}{N}\left(1-\frac{k}{N}\right)}\left\langle\sigma_{-}(\infty) \sigma_{+}(1) \mathcal{V}_{\mathrm{p}}(0)\right\rangle \\
& =N \int_{\Gamma} \frac{d^{2} p}{(2 \pi)^{2}} C_{-,+}^{\mathcal{V}}(\mathrm{p}) C_{\mathcal{V},-,+}(\mathrm{p})|x|^{2 \alpha^{\prime} \overline{\mathrm{p}}-\frac{2 k}{N}\left(1-\frac{k}{N}\right)} \\
& =V_{D-2}|x|^{-\frac{2 k}{N}\left(1-\frac{k}{N}\right)} \int \frac{d^{2} p}{(2 \pi)^{2}}\left|\frac{x}{\delta^{\prime}}\right|^{2 \alpha^{\prime} \overline{\mathrm{p}}} \\
& =\frac{1}{2 \pi \alpha^{\prime}}\left(-\ln \left|\frac{x}{\delta^{\prime}}\right|\right)^{-1}|x|^{-\frac{2 k}{N}\left(1-\frac{k}{N}\right)} V_{D-2}
\end{aligned}
$$

On the other hand, for $x \sim 0, F(x) \sim 1$ and $F(1-x) \sim \frac{1}{\pi} \sin \left(\frac{\pi k}{N}\right)\left(-\ln \frac{x}{\delta}\right)$. Here $\delta$ is a function of $k / n$ : $\ln \delta(k / N)=2 \psi(1)-\psi(k / N)-\psi(1-(k / N))$, where $\psi(x)=\Gamma^{\prime}(x) / \Gamma(x)$. In particular, $\delta(1 / 2)=2^{4}$ and $\delta(1 / 3)=3^{3}$. We then find

$$
Z(x, \bar{x}) \sim \mathcal{N}\left[\frac{2}{\pi} \sin \left(\frac{\pi k}{N}\right)\left(-\ln \left|\frac{x}{\delta}\right|\right)\right]^{-1}|x|^{-\frac{2 k}{N}\left(1-\frac{k}{N}\right)} .
$$

By comparing the two expressions of $Z(x, \bar{x})$ when $x \sim 0$, we deduce that

$$
\delta^{\prime}=\delta
$$


which fixes the three-point functions in (3.19). The value of $\mathcal{N}$ is also determined, and the result for $Z(x, \bar{x})$ is

$$
Z(x, \bar{x})=\frac{1}{\pi^{2} \alpha^{\prime}} \sin \left(\frac{\pi k}{N}\right) \frac{|x(1-x)|^{-\frac{2 k}{N}\left(1-\frac{k}{N}\right)}}{F(x) F(1-\bar{x})+F(1-x) F(\bar{x})} V_{D-2} .
$$

We now use the $x \rightarrow \infty$ behavior of $Z(x, \bar{x})$ to calculate the three-twist correlators

$$
C_{--,+,+}=\left\langle\sigma_{--}(\infty) \sigma_{+}(1) \sigma_{+}(0)\right\rangle, \quad C_{++,-,-}=\left\langle\sigma_{++}(\infty) \sigma_{-}(1) \sigma_{-}(0)\right\rangle .
$$

The coefficients $C_{+,+}^{++}$and $C_{-,-}^{--}$are defined by the leading terms in the OPE's,

$$
\sigma_{+}(x, \bar{x}) \sigma_{+}(0) \sim C_{+,+}^{++}|x|^{-\frac{2 k^{2}}{N^{2}}} \sigma_{++}(0), \quad \sigma_{-}(x, \bar{x}) \sigma_{-}(0) \sim C_{-,-}^{--}|x|^{-\frac{2 k^{2}}{N^{2}}} \sigma_{--}(0),
$$

and they and the three-twist correlators obey the following relations:

$$
C_{--,+,+}=C_{++,-,-}=C_{+,+}^{++} V_{D-2}=C_{-,-}^{--} V_{D-2} .
$$

On the one hand, for $x \rightarrow \infty$, we find

$$
Z(x, \bar{x}) \sim C_{-,-}^{--}|x|^{-\frac{2 k}{N}\left(1-\frac{2 k}{N}\right)}\left\langle\sigma_{--}(\infty) \sigma_{+}(1) \sigma_{+}(0)\right\rangle=C_{-,-}^{--} C_{--,+,+}|x|^{-\frac{2 k}{N}\left(1-\frac{2 k}{N}\right)} .
$$

On the other hand, for $x \rightarrow \infty$ the asymptotic forms of $F(x)$ and $F(1-x)$

$$
F(x) \sim \alpha^{\frac{k}{2}} \frac{\Gamma\left(1-\frac{2 k}{N}\right)}{\Gamma^{2}\left(1-\frac{k}{N}\right)} x^{-\frac{k}{N}}, \quad F(1-x) \sim \frac{\Gamma\left(1-\frac{2 k}{N}\right)}{\Gamma^{2}\left(1-\frac{k}{N}\right)} x^{-\frac{k}{N}}, \quad k / N<1 / 2,
$$

immediately give the $x \rightarrow \infty$ behavior of $Z(x, \bar{x})$ :

$$
Z(x, \bar{x}) \sim \frac{1}{2 \pi^{2} \alpha^{\prime}} \tan \left(\frac{\pi k}{N}\right) \frac{\Gamma^{4}\left(1-\frac{k}{N}\right)}{\Gamma^{2}\left(1-\frac{2 k}{N}\right)}|x|^{-\frac{2 k}{N}\left(1-\frac{2 k}{N}\right)} V_{D-2}, \quad k / N<1 / 2 .
$$

Comparing the two asymptotic formulae for $Z(x, \bar{x})$ given above, we find that

$$
C_{--,+,+}=C_{++,-,-}=\sqrt{\frac{1}{2 \pi^{2} \alpha^{\prime}} \tan \frac{\pi k}{N}} \frac{\Gamma^{2}\left(1-\frac{k}{N}\right)}{\Gamma\left(1-\frac{2 k}{N}\right)} V_{D-2}, \quad k / N<1 / 2 .
$$

This result can be compared with equation (4.47) of [31, which holds for an orbifold with finite twodimensional volume $V_{\Lambda}$. The answer in (3.31) is obtained by discarding the factor which involves the sum over classical solutions that exist for finite orbifolds and by replacing $V_{\Lambda}$ by $1 /\left(2 \pi^{2}\right)$. Additionally, (3.31) contains the $\alpha^{\prime}$ dependence necessary to get tachyon potentials with the correct units and the spacetime volume factor $V_{D-2}$. When the operators in any correlator that has this volume factor are dressed with $D-2$ dimensional momenta $q$, the factor must be replaced by $(2 \pi)^{D-2} \delta^{(D-2)}\left(\sum q\right)$.

\section{The $\mathbb{C} / \mathbb{Z}_{2}$ tachyon potential}

Having computed the necessary CFT correlators, we can formulate closed string field theory on orbifold backgrounds. In this section we study the string field theory of the simplest one, the $\mathbb{C} / \mathbb{Z}_{2}$ orbifold. We first compute the closed string field theory action, and then we carry out the level-truncation analysis. 


\subsection{Computing the action}

The $\mathbb{C} / \mathbb{Z}_{2}$ orbifold consists of two sectors: an untwisted sector corresponding to $k=0$ and a twisted sector with $k=1$. The structure of the untwisted sector is universal for any $\mathbb{C} / \mathbb{Z}_{N}$ orbifold. The vertex operator for the bulk tachyon is given by (3.12) and we write the corresponding string field as

$$
|U\rangle=\int \frac{d^{D-2} q}{(2 \pi)^{D-2}} \int \frac{d^{2} p}{(2 \pi)^{2}} u(q, p) c_{1} \bar{c}_{1}|q, p\rangle .
$$

Since the correlators of $c \bar{c} e^{i q \cdot X} \mathcal{U}_{\mathrm{p}}$ are identical to those of $c \bar{c} e^{i q \cdot X} e^{i p_{1} X^{1}+i p_{2} X^{2}}$ in the flat-space theory, the tachyon string field action takes the same form as (2.21):

$$
\begin{array}{r}
-\frac{1}{2} \int \frac{d^{D-2} q}{(2 \pi)^{D-2}} \int \frac{d^{2} p}{(2 \pi)^{2}} u(-q,-p)\left(q^{2}+p^{2}-\frac{4}{\alpha^{\prime}}\right) u(q, p) \\
-\frac{1}{3 !} \frac{4 \kappa}{\alpha^{\prime}} \int \prod_{i=1}^{3}\left[\frac{d^{D-2} q_{i}}{(2 \pi)^{D-2}} \frac{d^{2} p_{i}}{(2 \pi)^{2}} \mathcal{R}^{2-\frac{1}{2} \alpha^{\prime} q_{i}^{2}-\frac{1}{2} \alpha^{\prime} p_{i}^{2}} u\left(q_{i}, p_{i}\right)\right] \\
\times(2 \pi)^{D} \delta^{(D-2)}\left(q_{1}+q_{2}+q_{3}\right) \delta^{(2)}\left(p_{1}+p_{2}+p_{3}\right) .
\end{array}
$$

The only $N$ dependence that remains in the above terms is due to the condition $u(q, \mathrm{p})=u\left(q, e^{2 \pi i / N} \mathrm{p}\right)$. It is convenient to make a Fourier transformation on each of the flat $D-2$ directions:

$$
\begin{aligned}
& -\frac{1}{2} \int d^{D-2} x \int \frac{d^{2} p}{(2 \pi)^{2}}\left[\eta^{\mu \nu} \partial_{\mu} u(x,-p) \partial_{\nu} u(x, p)+u(x,-p)\left(p^{2}-\frac{4}{\alpha^{\prime}}\right) u(x, p)\right] \\
& -\frac{1}{3 !} \frac{4 \kappa}{\alpha^{\prime}} \int d^{D-2} x \int \prod_{i=1}^{3}\left[\frac{d^{2} p_{i}}{(2 \pi)^{2}} \mathcal{R}^{2+\frac{1}{2} \alpha^{\prime} \partial^{2}-\frac{1}{2} \alpha^{\prime} p_{i}^{2}} u\left(x, p_{i}\right)\right](2 \pi)^{2} \delta^{(2)}\left(p_{1}+p_{2}+p_{3}\right) .
\end{aligned}
$$

For configurations that are translationally invariant along the $(D-2)$-dimensional spacetime we can define a potential $\mathbb{V}$ that is equal to minus the $(D-2)$-dimensional Lagrangian density. The untwisted tachyon contribution to the potential is therefore

$$
\begin{aligned}
\mathbb{V}_{u^{2}}+\mathbb{V}_{u^{3}}= & -\frac{1}{2} \int \frac{d^{2} p}{(2 \pi)^{2}} u(-p)\left(\frac{4}{\alpha^{\prime}}-p^{2}\right) u(p) \\
& +\frac{1}{3 !} \frac{4 \kappa}{\alpha^{\prime}} \int \prod_{i=1}^{3}\left[\frac{d^{2} p_{i}}{(2 \pi)^{2}} \mathcal{R}^{2-\frac{1}{2} \alpha^{\prime} p_{i}^{2}} u\left(p_{i}\right)\right](2 \pi)^{2} \delta^{(2)}\left(p_{1}+p_{2}+p_{3}\right) .
\end{aligned}
$$

Let us next consider the twisted sector. The twist operator, denoted by $\sigma$, has conformal dimensions $h_{\sigma}=\bar{h}_{\sigma}=1 / 8$. The associated twisted tachyon field $t(x)$ has mass-squared equal to $2 / \alpha^{\prime}$ times the sum of the holomorphic and antiholomorphic dimensions of $c_{1} \bar{c}_{1}|\sigma\rangle: m^{2}=\frac{2}{\alpha^{\prime}}\left(-1+\frac{1}{8}-1+\frac{1}{8}\right)=\frac{4}{\alpha^{\prime}}\left(-\frac{7}{8}\right)$. The level of this field is obtained using (2.27):

$$
\ell(t)=\frac{1}{4}
$$

The next excited states in the twisted sector contain two matter oscillators each of which contributes level $1 / 2$, so the level of the corresponding tachyon states is $5 / 4$. Our present analysis will not include 
these states, whose quadratic terms appear at level 10/4, but only the bulk tachyon and the twisted tachyon $t$. The string field associated with $t$ is written as

$$
|T\rangle=\int \frac{d^{D-2} q}{(2 \pi)^{D-2}} t(q) c_{1} \bar{c}_{1}|\sigma, q\rangle, \quad t(q)=\int d^{D-2} x t(x) e^{-i q \cdot x} .
$$

Since both BPZ and Hermitian conjugations take $|\sigma\rangle$ to the same bra $\langle\sigma|$, the string field is real if $t(x)$ is real. The inner product is given by

$$
\begin{aligned}
\left\langle\sigma,-q^{\prime}\left|c_{-1} \bar{c}_{-1} \quad c_{0}^{-} c_{0}^{+} c_{1} \bar{c}_{1}\right| \sigma, q\right\rangle & =(2 \pi)^{D-2} \delta^{(D-2)}\left(q+q^{\prime}\right), \\
\left\langle\sigma,-q^{\prime}|c \bar{c}(\infty) c \bar{c}(1) c \bar{c}(0)| \sigma, q\right\rangle & =2(2 \pi)^{D-2} \delta^{(D-2)}\left(q+q^{\prime}\right),
\end{aligned}
$$

as expected for a twisted field that lives in $D-2$ spacetime dimensions. The kinetic term for the twisted tachyons is found to be

$$
-\frac{1}{2} \int \frac{d^{D-2} q}{(2 \pi)^{D-2}} t(-q)\left(q^{2}-\frac{4}{\alpha^{\prime}} \frac{7}{8}\right) t(q) .
$$

The associated contribution to the potential is

$$
\mathbb{V}_{t^{2}}=-\frac{1}{2} \frac{4}{\alpha^{\prime}} \frac{7}{8} t^{2}
$$

There is no $t^{3}$ interaction because of the $\mathbb{Z}_{2}$ symmetry of the orbifold CFT. There is, however, a cubic coupling that involves a bulk tachyon and two twisted tachyons. Using the formula (2.17) this interaction is given by the following term in the action:

$$
\begin{aligned}
& -\frac{1}{3 !} \frac{2 \kappa}{\alpha^{\prime}} \cdot 3 \cdot \int \prod_{i=1}^{3}\left[\frac{d^{D-2} q_{i}}{(2 \pi)^{D-2}} \mathcal{R}^{2-\frac{1}{2} \alpha^{\prime} q_{i}^{2}}\right] \int \frac{d^{2} p}{(2 \pi)^{2}} \mathcal{R}^{-\frac{1}{2} \alpha^{\prime} p^{2}} u\left(q_{1}, p\right) \mathcal{R}^{-\frac{1}{4}} t\left(q_{2}\right) \mathcal{R}^{-\frac{1}{4}} t\left(q_{3}\right) \\
& \times 2 \delta^{-\frac{1}{4} \alpha^{\prime} p^{2}}(2 \pi)^{D-2} \delta^{(D-2)}\left(q_{1}+q_{2}+q_{3}\right),
\end{aligned}
$$

where the factor on the second line is the three-point correlator $C_{\mathcal{V},-,+}(\mathrm{p})$ in (3.19), extended to the case where the operators are dressed with $(D-2)$-dimensional momenta and the ghosts are included. After Fourier transformation and simplification, the above term becomes

$$
-\frac{2 \kappa}{\alpha^{\prime}} \mathcal{R}^{\frac{11}{2}} \int d^{D-2} x \int \frac{d^{2} p}{(2 \pi)^{2}}\left(\mathcal{R}^{2} \delta\right)^{-\frac{1}{4} \alpha^{\prime} p^{2}}\left[\mathcal{R}^{\frac{\alpha^{\prime} \partial^{2}}{2}} u(x, p)\right]\left[\mathcal{R}^{\frac{\alpha^{\prime} \partial^{2}}{2}} t(x)\right]^{2} .
$$

The contribution to the potential from this coupling is

$$
\mathbb{V}_{u t^{2}}=\frac{2 \kappa}{\alpha^{\prime}} \mathcal{R}^{\frac{11}{2}} t^{2} \int \frac{d^{2} p}{(2 \pi)^{2}}\left(\mathcal{R}^{2} \delta\right)^{-\frac{1}{4} \alpha^{\prime} p^{2}} u(p), \quad \delta=2^{4} \text { for } \mathbb{C} / \mathbb{Z}_{2}
$$

Collecting all the contributions, the potential is given by

$$
\begin{aligned}
\mathbb{V}_{2}= & -\frac{1}{2} \frac{4}{\alpha^{\prime}} \frac{7}{8} t^{2}-\frac{1}{2} \int \frac{d^{2} p}{(2 \pi)^{2}} u(-p)\left(\frac{4}{\alpha^{\prime}}-p^{2}\right) u(p) \\
& +\frac{2 \kappa}{\alpha^{\prime}} \mathcal{R}^{\frac{11}{2}} t^{2} \int \frac{d^{2} p}{(2 \pi)^{2}}\left(\mathcal{R}^{2} \delta\right)^{-\frac{1}{4} \alpha^{\prime} p^{2}} u(p) \\
& +\frac{1}{3 !} \frac{4 \kappa}{\alpha^{\prime}} \int \prod_{i=1}^{3}\left[\frac{d^{2} p_{i}}{(2 \pi)^{2}} \mathcal{R}^{2-\frac{1}{2} \alpha^{\prime} p_{i}^{2}} u\left(p_{i}\right)\right](2 \pi)^{2} \delta^{(2)}\left(p_{1}+p_{2}+p_{3}\right) .
\end{aligned}
$$


In order to eliminate $\alpha^{\prime}$ and $\kappa$ we define dimensionless momentum variables $\left(\xi_{1}, \xi_{2}\right)$ and conjugate coordinates $\left(r_{1}, r_{2}\right)$ by

$$
p_{a}=\frac{2}{\sqrt{\alpha^{\prime}}} \xi_{a}, \quad x_{a}=\frac{\sqrt{\alpha^{\prime}}}{2} r_{a}, \quad a=1,2,
$$

where $x_{a}$ is the coordinate conjugate to $p_{a}$. Additionally, we define

$$
u(\xi)=\frac{4 \kappa}{\alpha^{\prime}} u(p)
$$

and rescale $t \rightarrow \frac{\sqrt{\alpha^{\prime}}}{\kappa} t$. The resulting expression for the ratio $f_{2}$ defined by (2.9) is

$$
\begin{aligned}
f_{2}=\frac{\kappa^{2} \mathbb{V}_{2}}{\pi}= & -\frac{7}{4 \pi} t^{2}-\frac{1}{2 \pi} \int \frac{d^{2} \xi}{(2 \pi)^{2}} u(-\xi)\left(1-\xi^{2}\right) u(\xi) \\
& +\frac{2}{\pi} \mathcal{R}^{\frac{11}{2}} t^{2} \int \frac{d^{2} \xi}{(2 \pi)^{2}}\left(\mathcal{R}^{2} \delta\right)^{-\xi^{2}} u(\xi) \\
& +\frac{1}{6 \pi} \int \prod_{i=1}^{3}\left[\frac{d^{2} \xi_{i}}{(2 \pi)^{2}} \mathcal{R}^{2\left(1-\xi_{i}^{2}\right)} u\left(\xi_{i}\right)\right](2 \pi)^{2} \delta^{(2)}\left(\xi_{1}+\xi_{2}+\xi_{3}\right) .
\end{aligned}
$$

We will be analyzing $f_{2}$ in the level expansion. The level of the untwisted tachyon $u(p)$ is given by (2.28):

$$
\ell(u(p))=\frac{1}{2} \alpha^{\prime} p^{2}=2 \xi^{2} .
$$

Working up to some level means including only a limited set of momentum modes of the field $u(p)$. This is exactly the way that open string tachyon modes were treated in the case of spatially dependent lump solutions [14. As we discussed earlier, the level of the twisted field $t$ is equal to $1 / 4$, so it is reasonable to use all bulk tachyon modes that have level less than or equal to $1 / 4$. We therefore demand

$$
\ell(u(\xi))=2 \xi^{2} \leq \frac{1}{4} \quad \rightarrow \quad|\xi| \leq \xi_{*}=\sqrt{\frac{1}{8}} \simeq 0.354
$$

As a result, in the level expansion analysis of $f_{2}$, the integrals must be cutoff at $\xi=\xi_{*}$. The levels of the various fields and interactions are represented in Fig. 1. With maximum level $1 / 4$ for $u(p)$, the cubic self-interaction $u^{3}$ has level less than or equal to $3 / 4$. For illustration purposes only, this figure includes couplings of the excited twisted tachyons $t_{\mathrm{ex}}$ that appear at levels higher than the cutoff level $3 / 4$.

To study $f_{2}$ using coordinate space we define $u(r)$ by standard two-dimensional Fourier transformation:

$$
u(\xi)=\int d^{2} r u(r) e^{-i \xi \cdot r} .
$$

The relation between $u(p)$ and $u(x)$ in (2.20) together with (4.15), (4.16), and (4.20) implies that $u(x)=u(r) / \kappa$. We use (4.20) and the rescaled $t$ to rewrite $f_{2}$ in coordinate space. Since we are only interested in rotationally invariant configurations of the field $u$, we find

$$
f_{2}=-\frac{7}{4 \pi} t^{2}+\int_{0}^{\infty} r d r\left(-u(r)\left(\partial^{2}+1\right) u(r)+\frac{\mathcal{R}^{\frac{11}{2}} t^{2}}{\pi \ln \left(\mathcal{R}^{2} \delta\right)} e^{-\frac{r^{2}}{4 \ln \left(\mathcal{R}^{2} \delta\right)}} u(r)+\frac{1}{3}\left[\mathcal{R}^{2\left(\partial^{2}+1\right)} u(r)\right]^{3}\right)
$$




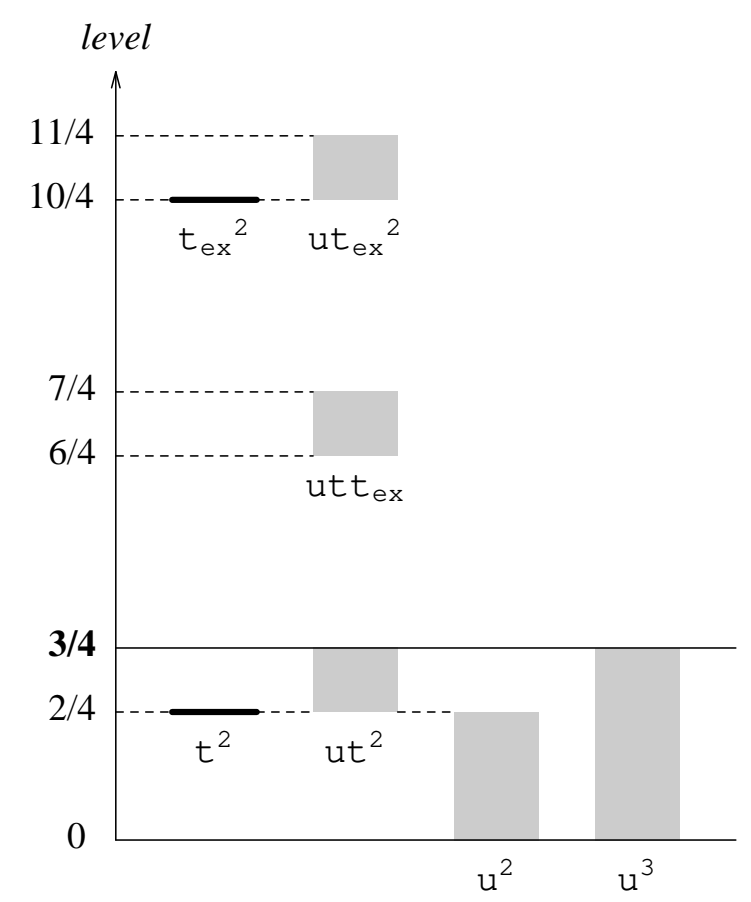

Figure 1: Level expansion table for the $\mathbb{C} / \mathbb{Z}_{2}$ orbifold. Since the bulk tachyon includes all levels from zero to $1 / 4$, interactions that involve the bulk tachyon are shown as bands. Terms involving excited twisted tachyons $t_{\text {ex }}$ are shown, but they are not included in our analysis.

where $r=\sqrt{r_{1}^{2}+r_{2}^{2}}$, and $\partial^{2}=\frac{\partial^{2}}{\partial r^{2}}+\frac{1}{r} \frac{\partial}{\partial r}$ is the two-dimensional Laplacian that acts on rotationally invariant functions. Equation (4.21) will be the starting point of our coordinate space analysis.

\subsection{A preliminary analysis}

We have presented an expression (4.17) for $f_{2}$ that incorporates a restricted set of interactions. We must now find the critical points of $f_{2}$ in the configuration space of $t$ and $u(\xi)$. If the value of $f_{2}$ at a critical point is near minus one, this can be taken as evidence for the conjecture that gives the height of the potential in terms of the deficit angle of the cone.

The equations that define critical points are made very nontrivial by the cubic term of the bulk tachyon $u$. Let us first study $f_{2}$ in the approximation where the $u^{3}$ term is ignored. We will take $f_{2}$ to be given by

$$
f_{2}=-\frac{7}{4 \pi} t^{2}-\frac{1}{2 \pi} \int \frac{d^{2} \xi}{(2 \pi)^{2}} u(-\xi)\left(1-\xi^{2}\right) u(\xi)+\frac{2}{\pi} \mathcal{R}^{\frac{11}{2}} t^{2} \int \frac{d^{2} \xi}{(2 \pi)^{2}}\left(\mathcal{R}^{2} \delta\right)^{-\xi^{2}} u(\xi),
$$

and we will determine the critical points of this potential analytically.

It is in fact instructive to obtain an effective potential for $t$ by solving for $u(\xi)$ in terms of $t$ and substituting back into $f_{2}$. Varying $f_{2}$ with respect to $u(\xi)$ one readily finds that

$$
u(\xi)=2 \mathcal{R}^{\frac{11}{2}} \frac{\left(\mathcal{R}^{2} \delta\right)^{-\xi^{2}}}{1-\xi^{2}} t^{2}
$$


Substituting back into (4.22), the effective potential for $t$ acquires a positive $t^{4}$ term:

$$
f_{2}=-\frac{7}{4 \pi} t^{2}+\frac{2}{\pi} \eta\left(\mathcal{R}, \delta, \xi_{*}\right) \mathcal{R}^{11} t^{4}
$$

where $\eta\left(\mathcal{R}, \delta, \xi_{*}\right)$ is a constant defined by the following integral: ${ }^{7}$

$$
\eta\left(\mathcal{R}, \delta, \xi_{*}\right)=\int_{0}^{\xi_{*}} d \xi \frac{\xi}{2 \pi} \frac{\left(\mathcal{R}^{2} \delta\right)^{-2 \xi^{2}}}{1-\xi^{2}} \simeq 0.00717348,
$$

evaluated with $\delta=2^{4}$ and $\xi_{*}=\sqrt{1 / 8}$. While $\mathcal{R}$ is a constant, we include it as an argument of $\eta$ to discuss the $\mathcal{R}$ dependence of the results explicitly. The potential (4.24) has two critical points with the same value of $f_{2}$ :

$$
f_{2}=-\frac{49}{128 \pi} \frac{\mathcal{R}^{-11}}{\eta\left(\mathcal{R}, \delta, \xi_{*}\right)} \simeq-0.955577 .
$$

This answer is remarkably close to the expected value! We will see in the following subsections that the inclusion of the cubic bulk tachyon term modifies the result, but not drastically. The cubic term must be included, of course, in any systematic analysis that aims to be fully quantitative.

Had we not used the closed string field theory action, but rather the naive extrapolation from on-shell CFT computations, $f_{2}$ would be given by the expression in (4.26) evaluated for $\mathcal{R}=1$. It turns out that $\eta$ is a relatively slowly varying function of $\mathcal{R}$ : for $\mathcal{R}=1, \eta \simeq 0.00760665$, which is about $6 \%$ larger than the value given in (4.25). Therefore the change in $f_{2}$ is mostly due to the factor $\mathcal{R}^{-11} \simeq 1 / 17.78$. Indeed, for $\mathcal{R}=1$, one finds $f_{2} \simeq-16.0193$, which is far away from the expected result. This demonstrates that quantitative study requires the use of the off-shell CSFT action.

This value of $f_{2}$ does not change much when we increase the momentum cutoff $\xi_{*}$. Increasing the cutoff is equivalent to increasing the level $\ell$ of the allowed bulk tachyon modes. It is clear from Fig. 1] that it is not really consistent to allow arbitrarily high-level tachyon modes without including other fields and interactions. We do it, however, to get a sense of the effects of higher bulk tachyon modes. While $\xi_{*}$ is less than one and increasing, $\eta$ increases, the quartic term becomes larger, and the critical point becomes shallower. If $\xi_{*}>1$, which corresponds to level $\ell>2$, the integral for $\eta$ requires a prescription to deal with the pole at $\xi=1$. If we take the principal value at $\xi=1$, the value of $f_{2}$ remains essentially constant for all $\xi_{*}>1$ and $f_{2} \simeq-0.456693$ in the limit $\xi_{*} \rightarrow \infty$. The singularity at $\xi=1$ arises because the bulk tachyon becomes marginal at this momentum. The coordinate space solution develops a long-ranged Bessel function tail, and a detailed study shows that the principal value prescription gives a solution with minimal tail.

\subsection{Level expansion analysis}

We now investigate the critical points of the functional $f_{2}$ including the cubic term of the bulk tachyon. It is no longer possible to solve the equations of motion analytically, so we discretize the spectrum of the bulk tachyon and solve the equations numerically. We explore two different methods for the

\footnotetext{
${ }^{7}$ Actually, $\eta\left(\mathcal{R}, \delta, \xi_{*}\right)=\left(4 \pi \mathcal{R}^{4} \delta^{2}\right)^{-1}\left[\operatorname{Ei}\left(2 \ln \left(\mathcal{R}^{2} \delta\right)\right)-\operatorname{Ei}\left(2\left(1-\xi_{*}^{2}\right) \ln \left(\mathcal{R}^{2} \delta\right)\right)\right]$, where the exponential integral function $\operatorname{Ei}(x)=-\int_{-x}^{\infty} d t \frac{e^{-t}}{t}$ is defined by taking the principal value at $t=0$.
} 
discretization, in both of which the level is constrained as in (4.19). In the first one we use coordinate space: we replace the two-dimensional space of the cone by a round disk and impose the condition that the bulk tachyon vanishes at the boundary. In the second one we do a straightforward discretization of the momentum space representation of $f_{2}$. By doing so, the two-dimensional space of the cone has been replaced by a square box with periodic boundary conditions. Since we are looking for a solution where the bulk tachyon is localized near the origin, we expect both methods to give the same result. Indeed, this is what we find.

\subsubsection{Coordinate space method}

In this approach we study $f_{2}$ (see (4.21) ) by imposing the condition $u(r=L)=0$, where $L$ is a length to be fixed by the desired accuracy of the analysis. We will expand $u(r)$ using Bessel functions $J_{0}(\xi r)$ that vanish for $r=L$. We can view $J_{0}(\xi r)$ as a superposition of plane waves,

$$
J_{0}(\xi r)=\frac{1}{2 \pi} \int_{0}^{2 \pi} d \theta e^{i \xi r \cos \theta}
$$

each of which carries momentum of magnitude $\xi$. Indeed, the two-dimensional Laplacian gives $\partial^{2} J_{0}(\xi r)=-\xi^{2} J_{0}(\xi r)$. We therefore expand the bulk tachyon $u(r)$ in terms of functions $J_{0}(\xi r)$ with $\xi \leq \xi_{*}$. We can select $n$ such functions

$$
\left\{J_{0}\left(\xi_{1} r\right), J_{0}\left(\xi_{2} r\right), \ldots J_{0}\left(\xi_{n} r\right)\right\} \quad \text { with } \quad \xi_{1}<\xi_{2}<\ldots<\xi_{n}=\xi_{*},
$$

by imposing the $n$ conditions $\xi_{i} L=\alpha_{i}$, where $\alpha_{i}$ denotes the $i$-th zero of $J_{0}(x): J_{0}\left(\alpha_{i}\right)=0$. We use

the condition with $i=n$ to fix the size $L$ of the disk and the remaining conditions to fix the values of $\xi_{i}$ :

$$
L=\frac{\alpha_{n}}{\xi_{*}}, \quad \xi_{i}=\frac{\alpha_{i}}{L}=\frac{\alpha_{i}}{\alpha_{n}} \xi_{*} .
$$

The bulk tachyon $u(r)$ is thus expanded as

$$
u(r)=\sum_{i=1}^{n} u_{i} \psi_{i}(r), \quad \text { with } \quad \psi_{i}(r)=\frac{\xi_{*}}{\sqrt{\pi} \alpha_{n} J_{1}\left(\alpha_{i}\right)} J_{0}\left(\alpha_{i} \frac{r}{L}\right),
$$

which are functions normalized to satisfy

$$
2 \pi \int_{0}^{L} d r r \psi_{i}(r) \psi_{j}(r)=\delta_{i j}
$$

At this $n$-th order approximation, $f_{2}$ is a function of the $n$ modes $u_{i}$ of the bulk tachyon and the twisted tachyon $t$. The continuum spectrum is recovered in the limit $n \rightarrow \infty$.

Let us now turn to the explicit computations. Many of the general features of the minimization of $f_{2}$ can be gleaned from the simplest case $n=1$, which we explore first. Although the resulting values of $f_{2}$ differ substantially from those obtained for large $n$, the $n=1$ case can be studied analytically. We then turn to the large $n$ numerical analysis. 
Lowest-order approximation. A simple computation shows that for $n=1, f_{2}$ is given by

$$
f_{2}=-\frac{1}{2} m_{u}^{2} u_{1}^{2}-\frac{1}{2} m_{t}^{2} t^{2}+g_{u^{3}} u_{1}^{3}+g_{u t^{2}} u_{1} t^{2},
$$

where

$$
\begin{aligned}
& m_{u}^{2}=\frac{1-\xi_{*}^{2}}{\pi} \simeq 0.278521, \quad m_{t}^{2}=\frac{7}{2 \pi} \simeq 1.11408 \\
& g_{u^{3}}=\frac{\xi_{*} \mathcal{R}^{6\left(1-\xi_{*}^{2}\right)}}{3 \pi^{\frac{3}{2}} \alpha_{1} J_{1}\left(\alpha_{1}\right)^{3}} \int_{0}^{1} d y y J_{0}\left(\alpha_{1} y\right)^{3} \simeq 0.0242103 \\
& g_{u t^{2}}=\frac{\alpha_{1} \mathcal{R}^{\frac{11}{2}}}{\pi^{\frac{3}{2}} \xi_{*} J_{1}\left(\alpha_{1}\right) \ln \left(\mathcal{R}^{2} \delta\right)} \int_{0}^{1} d y y e^{-\frac{\alpha_{1}^{2}}{4 \xi_{*}^{2} \ln \left(\mathcal{R}^{2} \delta\right)} y^{2}} J_{0}\left(\alpha_{1} y\right) \simeq 0.285768 .
\end{aligned}
$$

Although $f_{2}$ contains four parameters, we can eliminate two of them by rescaling $u_{1}$ and $t$, and we can eliminate a third one by rescaling $f_{2}$ itself:

$$
\widetilde{f}_{2}=-\frac{1}{2} \tilde{u}_{1}^{2}-\frac{1}{2} \tilde{t}^{2}+\frac{g}{3} \tilde{u}_{1}^{3}+\tilde{u}_{1} \tilde{t}^{2}
$$

where

$$
\tilde{f}_{2}=\frac{g_{u t^{2}}^{2}}{m_{u}^{2} m_{t}^{4}} f_{2}, \quad \tilde{u}_{1}=\frac{g_{u t^{2}}}{m_{t}^{2}} u_{1}, \quad \tilde{t}=\frac{g_{u t^{2}}}{m_{u} m_{t}} t, \quad g=\frac{3 g_{u^{3}} m_{t}^{2}}{g_{u t^{2}} m_{u}^{2}} .
$$

This form is useful because the vacuum structure is governed only by $g$, which in our case is given by

$$
g \simeq 1.01664
$$

Solving the equations $\partial \widetilde{f}_{2} / \partial \tilde{u}_{1}=\partial \widetilde{f}_{2} / \partial \tilde{t}=0$, we find three nontrivial critical points:

$$
\begin{aligned}
& \tilde{f}_{2}=-\frac{3-g}{24} \quad \text { for } \quad\left(\tilde{u}_{1}, \tilde{t}\right)=\left(\frac{1}{2}, \pm \frac{\sqrt{2-g}}{2}\right), \\
& \tilde{f}_{2}=-\frac{1}{6 g^{2}} \quad \text { for } \quad\left(\tilde{u}_{1}, \tilde{t}\right)=\left(\frac{1}{g}, 0\right) .
\end{aligned}
$$

When $g>2$, the two solutions with nonvanishing $\tilde{t}$ disappear, but we have all of the three critical points (see (4.36) $)$. In the original set of variables $\left(u_{1}, t\right)$, the critical points and the values of $f_{2}$ are given by

$$
\begin{array}{ll}
\left(u_{1}, t\right) \simeq(1.94928, \pm 0.966497), & f_{2} \simeq-0.349830, \\
\left(u_{1}, t\right) \simeq(3.83475,0), & f_{2} \simeq-0.682622 .
\end{array}
$$

We are interested in the critical points with a nonvanishing $t$, which appear in pairs with opposite signs due to the $\mathbb{Z}_{2}$ symmetry. The value of $f_{2}$ at these critical points is approximately $35 \%$ of the predicted value. It is encouraging that this coarse $n=1$ approximation gave a potential which is of the same order with the predicted value. As we shall see, the result improves quickly for $n>1$. 

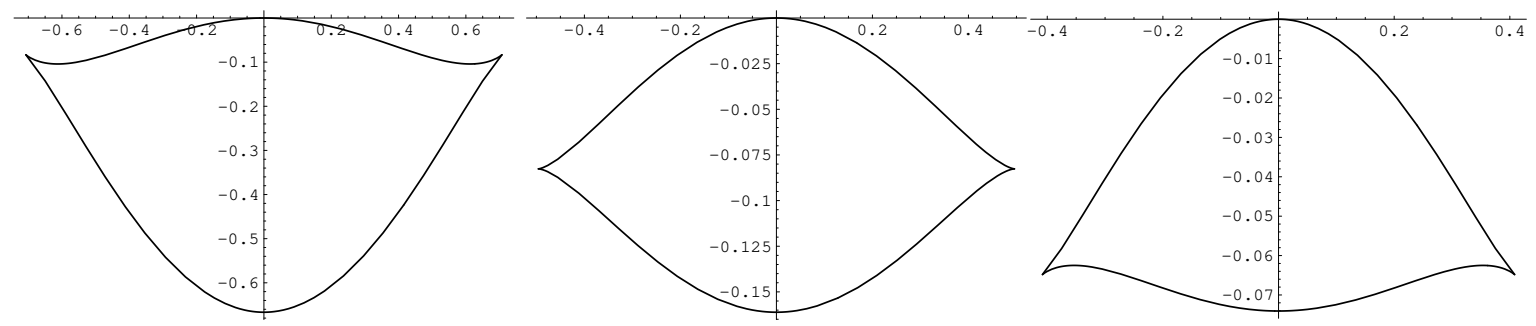

Figure 2: Effective potential $\widetilde{f}_{\text {eff }}(\tilde{t})$ for $g=0.5$ (left), $g=1.017$ (center), and $g=1.5$ (right). The two branches of the effective potential meet at cusps located at $\tilde{t}= \pm 1 /(2 \sqrt{g})$. The critical points lie on the upper branch for $g=0.5$. They pass through the cusps at $g=1$ and move to the lower branch for $g>1$.

It is instructive to consider the effective twisted tachyon potential as a function of the parameter $g$. The equation of motion for $\tilde{u}_{1}$ gives

$$
\tilde{u}_{1}=\frac{1 \mp \sqrt{1-4 g \tilde{t}^{2}}}{2 g}
$$

Correspondingly, there are two branches of the effective potential, upper and lower:

$$
\widetilde{f}_{\text {eff }}=\frac{-1+6 g \tilde{t}^{2}-6 g^{2} \tilde{t}^{2}}{12 g^{2}} \pm \frac{\left(1-4 g \tilde{t}^{2}\right)^{\frac{3}{2}}}{12 g^{2}} .
$$

The critical points are determined by the equation

$$
\frac{\partial \tilde{f}_{\mathrm{eff}}}{\partial \tilde{t}}=\frac{\tilde{t}}{g}\left(1-g \mp \sqrt{1-4 g \tilde{t}^{2}}\right)=0 .
$$

The properties of $\widetilde{f}_{\text {eff }}$ are illustrated in Fig. 2, For $0<g<1$ the nontrivial critical points lie on the upper branch and are local minima. For $1<g<2$ the critical points lie on the lower branch and they are local maxima. Despite appearances, nothing dramatic occurs when $g$ goes through one. The full potential (4.34) can be expanded about the critical points using

$$
\tilde{u}_{1}=\frac{1}{2}+\delta \tilde{u}_{1}, \quad \tilde{t}= \pm \frac{\sqrt{2-g}}{2}+\delta \tilde{t}
$$

and the resulting quadratic terms are

$$
\begin{aligned}
& \frac{1}{2}(g-1) \delta \tilde{u}_{1}^{2} \pm \sqrt{2-g} \delta \tilde{u}_{1} \delta \tilde{t} \\
= & \frac{1}{2}\left(\frac{1}{\sqrt{3-g}} \delta \tilde{u}_{1} \pm \sqrt{\frac{2-g}{3-g}} \delta \tilde{t}\right)^{2}-\frac{1}{2}(2-g)\left(\mp \sqrt{\frac{2-g}{3-g}} \delta \tilde{u}_{1}+\frac{1}{\sqrt{3-g}} \delta \tilde{t}\right)^{2} .
\end{aligned}
$$

This quadratic form has one positive eigenvalue and one negative eigenvalue for the entire range $0<g<2$. We interpret this as condensation of the twisted tachyon, while the instability associated with the bulk tachyon still remains. We note that the mass eigenstates arise through large mixing between $\delta \tilde{u}_{1}$ and $\delta \tilde{t}$. The effective potential only captures one direction in the two-dimensional field space and the apparent mass at the critical point does not bear any simple relation to the mass 
eigenvalues of the full potential. In particular, the transition from $g<1$ to $g>1$ is completely regular.

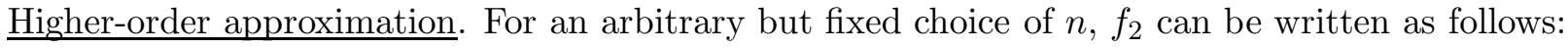

$$
f_{2}=-\frac{1}{2} \sum_{i=1}^{n} m_{i}^{2} u_{i}^{2}-\frac{1}{2} m_{t}^{2} t^{2}+\sum_{i, j, k=1}^{n} g_{i j k} u_{i} u_{j} u_{k}+\sum_{i=1}^{n} c_{i} u_{i} t^{2}
$$

where

$$
\begin{aligned}
m_{i}^{2} & =\frac{1}{\pi}\left(1-\frac{\alpha_{i}^{2}}{\alpha_{n}^{2}} \xi_{*}^{2}\right) \\
g_{i j k} & =\frac{\xi_{*} \mathcal{R}^{6-\frac{2 \xi_{*}^{2}}{\alpha_{n}^{2}}\left(\alpha_{i}^{2}+\alpha_{j}^{2}+\alpha_{k}^{2}\right)}}{3 \pi^{\frac{3}{2}} \alpha_{n} J_{1}\left(\alpha_{i}\right) J_{1}\left(\alpha_{j}\right) J_{1}\left(\alpha_{k}\right)} \int_{0}^{1} d y y J_{0}\left(\alpha_{i} y\right) J_{0}\left(\alpha_{j} y\right) J_{0}\left(\alpha_{k} y\right), \\
c_{i} & =\frac{\alpha_{n} \mathcal{R}^{\frac{11}{2}}}{\pi^{\frac{3}{2}} \xi_{*} J_{1}\left(\alpha_{i}\right) \ln \left(\mathcal{R}^{2} \delta\right)} \int_{0}^{1} d y y e^{-\frac{\alpha_{n}^{2}}{4 \xi_{*}^{2} \ln \left(\mathcal{R}^{2} \delta\right)} y^{2}} J_{0}\left(\alpha_{i} y\right) .
\end{aligned}
$$

We have carried out the numerical analysis up to $n=30$. As we increase $n$, we find a large number of critical points. Most of them have a large negative value for $f_{2}$, which seems to indicate that these solutions are not localized. For each $n$, however, there are three critical points on which $\left|f_{2}\right|$ remains small and which correspond to the three critical points we found for $n=1$. The critical points with $t \neq 0$ appear as pairs with opposite signs. These critical points are equivalent and the quadratic form of fluctuations about each of them has one positive and $n$ negative eigenvalues. This is consistent with our interpretation that the twisted tachyon disappears, while the instability associated with the bulk tachyon remains. For $t \neq 0$ the values of $f_{2}$ obtained for $n=16,17, \ldots, 30$ can be used to produce the approximate fit:

$$
f_{2}=-0.720205+\frac{1}{n} 0.522613 .
$$

This expression can be used to extrapolate $f_{2}$ in the limit $n \rightarrow \infty$ and gives $f_{2}=-0.720205$. Therefore, our level-truncation analysis gives approximately $72 \%$ of the predicted value for the potential depth.

The third critical point has vanishing $t$. We find a fit $f_{2}=-2.23467+2.18465 / n$. This is a pure bulk tachyon solution whose physical interpretation is not clear to us. We also do not know if this solution survives the inclusion of the quartic term in the bulk tachyon potential.

In Fig. 3 we show the coordinate-space profile $u(r)$ of the bulk tachyon, calculated with $n=30$. For this value of $n$ the cutoff radius of the cone, where the field $u(r)$ is required to vanish, is $r \simeq 264.36$. The figure illustrates that the bulk tachyon excitation is well localized near the apex of the cone. The cutoff is large enough to exhibit this localization and we have found that the profile remains essentially unchanged for larger $n$.

It is natural to wonder if the level of the bulk tachyon modes can be increased in a way consistent with a systematic level expansion. If we glance at Fig. 1 we see that a new interaction appears at level 6/4. It may therefore make sense to increase the level of the bulk tachyon field to $5 / 12$ so that the $u^{3}$ 


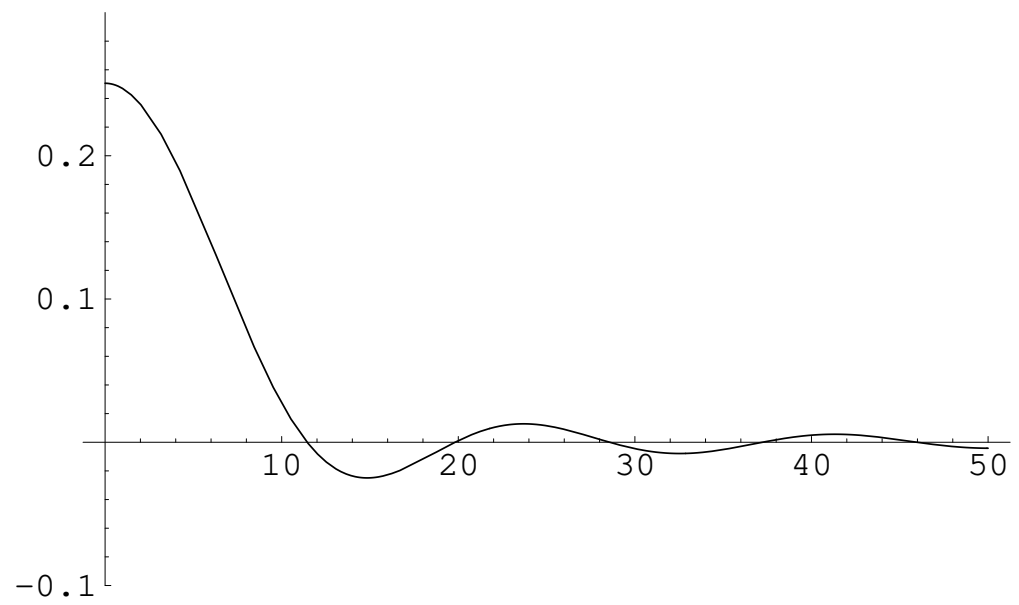

Figure 3: Coordinate-space profile $u(r)$ of the bulk tachyon for $n=30$. While $u(r)$ is required to vanish at $r=\alpha_{30} / \xi_{*} \simeq 264.36$, we see that it is localized in a much shorter range.

interaction carries level less than or equal to $5 / 4$. We have repeated the analysis of the potential for the corresponding new value of $\xi_{*}$ and find

$$
f_{2}=-0.503833+\frac{1}{n} 0.311575 \text { for } \quad \xi_{*}=\sqrt{5 / 24} .
$$

The extrapolation thus gives $f_{2} \simeq-0.504$, which is worse than the result obtained with lower momentum cutoff. Just as we saw in the preliminary analysis of 4.2 including higher level momentum modes of the bulk tachyon makes the critical point shallower. This result shows that the level expansion is not going to provide a monotonic approach to the expected value of $f_{2}$. It may be expected that as the level of interactions is increased and new fields appear, the value of $f_{2}$ will start to decrease.

\subsubsection{Momentum space method}

In this second approach, which we only sketch briefly, we simply discretize the integrals in the momentum space expression (4.17) for $f_{2}$. The discretization is characterized by an integer $N$. We discretize $\xi=\left(\xi_{1}, \xi_{2}\right)$ as

$$
\left(\xi_{1}, \xi_{2}\right)=\left(\frac{i}{N} \xi_{*}, \frac{j}{N} \xi_{*}\right), \quad \text { with } \quad-N \leq i, j \leq N .
$$

The allowed discrete values of the momenta form a square lattice. Since the smallest nonvanishing value of $\xi_{1}$ (or $\xi_{2}$ ) is $\xi_{*} / N$, the discretization amounts to placing the system in a two-dimensional square box of size $2 \pi N / \xi_{*}$ and imposing periodic boundary conditions.

The integrals can then be written in terms of finite sums that involve variables $u[i, j] \equiv u\left[\xi_{1}, \xi_{2}\right]$. The rotational symmetry of the problem allows us to reduce the number of variables significantly. Since $u[i, j]=u\left[i^{\prime}, j^{\prime}\right]$ whenever $i^{2}+j^{2}=i^{\prime 2}+j^{\prime 2}$, we can use a function of a single discrete variable by setting $u[i, j] \rightarrow u\left[i^{2}+j^{2}\right]$. For a fixed $N$, we further restrict the variables to $u\left[i^{2}+j^{2}\right]$ with $i^{2}+j^{2} \leq N$. In other words we use disks in momentum space. For $N=3$, for example, we have seven variables $u[0], u[1], u[2], u[4], u[5], u[8]$, and $u[9]$. 
Integrals are easily discretized as follows:

$$
\int \frac{d^{2} \xi}{(2 \pi)^{2}} F\left(\xi^{2}, u(\xi)\right) \simeq\left(\frac{\xi_{*}}{2 \pi N}\right)^{2} \sum_{i^{2}+j^{2} \leq N^{2}} F\left(\frac{\xi_{*}^{2}}{N^{2}}\left(i^{2}+j^{2}\right), u\left[i^{2}+j^{2}\right]\right) .
$$

The full expression for $f_{2}$, including multiple integrals, is readily discretized and we have determined the critical points of the potential for various values of $N$. Taking $\xi_{*}=\sqrt{1 / 8}$, and $N=3,4,5$, and 6 , we find $f_{2}=-0.710148,-0.735818,-0.706106$, and -0.721683 , respectively. For higher values of $N$, the solution exhibits decreasing oscillations about a value consistent with (4.50). Taking $\xi_{*}=\sqrt{5 / 24}$ we find values fully consistent with (4.51).

\section{$5 \quad$ The $\mathbb{C} / \mathbb{Z}_{3}$ tachyon potential}

The $\mathbb{C} / \mathbb{Z}_{3}$ orbifold has a tachyon potential that is more intricate than that of the $\mathbb{C} / \mathbb{Z}_{2}$ orbifold. First, the twisted tachyon is a complex field. Second, pure cubic interactions of the twisted tachyon exist. We find that the tachyon potential has two inequivalent critical points. In the deeper one, which we conjecture to represent flat space, two tachyonic modes are lifted. In the shallower one, which we conjecture to represent the $\mathbb{C} / \mathbb{Z}_{2}$ orbifold, one tachyonic mode is lifted.

\subsection{Computing the action}

In addition to the untwisted sector with $k=0$, the $\mathbb{C} / \mathbb{Z}_{3}$ orbifold includes two twisted sectors $k=1$ and $k=2$. The contributions to the potential from the untwisted sector are the same as those of $\mathbb{C} / \mathbb{Z}_{2}$ and are given by (4.4). There are a pair of twist fields $\sigma_{1}$ and $\sigma_{2}$, which we denote by $\sigma_{1}=\sigma_{+}=\sigma_{--}$ and $\sigma_{2}=\sigma_{-}=\sigma_{++}$following the conventions in $\$ 3$ Their conformal dimensions are $h_{\sigma_{ \pm}}=\bar{h}_{\sigma_{ \pm}}=1 / 9$. The mass-squared and the level of the corresponding spacetime field $t$ are

$$
m^{2}=\frac{4}{\alpha^{\prime}}\left(-\frac{8}{9}\right), \quad \ell(t)=\frac{2}{9} .
$$

The string field associated with the complex tachyon $t$ is written as

$$
|T\rangle=\int \frac{d^{D-2} q}{(2 \pi)^{D-2}}\left(t(q) c_{1} \bar{c}_{1}\left|\sigma_{+}, q\right\rangle+t^{*}(-q) c_{1} \bar{c}_{1}\left|\sigma_{-}, q\right\rangle\right), \quad t(q)=\int d^{D-2} x t(x) e^{-i q \cdot x}
$$

Since BPZ conjugation takes $\left|\sigma_{ \pm}\right\rangle$to $\left\langle\sigma_{ \pm}\right|$and Hermitian conjugation takes $\left|\sigma_{ \pm}\right\rangle$to $\left\langle\sigma_{\mp}\right|$, this is a real string field which leads to a real action. The inner product is given by

$$
\left\langle\sigma_{ \pm},-q^{\prime}\left|c_{-1} \bar{c}_{-1} c_{0}^{-} c_{0}^{+} c_{1} \bar{c}_{1}\right| \sigma_{\mp}, q\right\rangle=(2 \pi)^{D-2} \delta^{(D-2)}\left(q+q^{\prime}\right)
$$

and the kinetic term for the twisted tachyon and its contribution to the potential are found to be

$$
-\int \frac{d^{D-2} q}{(2 \pi)^{D-2}} t^{*}(q)\left(q^{2}-\frac{4}{\alpha^{\prime}} \frac{8}{9}\right) t(q), \quad \mathbb{V}_{t^{*} t}=-\frac{4}{\alpha^{\prime}} \frac{8}{9} t^{*} t
$$


Cubic interactions of the twisted tachyon are allowed by the $\mathbb{Z}_{3}$ symmetry. The three-point function is given by (3.31) with $k / N=1 / 3$ :

$$
C_{+,+,+}=\frac{3^{1 / 4}}{\sqrt{2 \pi^{2} \alpha^{\prime}}} \frac{\Gamma^{2}(2 / 3)}{\Gamma(1 / 3)} V_{D-2}=\frac{3^{3 / 4} \Gamma^{3}(2 / 3)}{2 \sqrt{2 \alpha^{\prime}} \pi^{2}} V_{D-2} .
$$

The contribution to the potential from the cubic coupling is given by

$$
\mathbb{V}_{t^{3}+\left(t^{*}\right)^{3}}=\frac{1}{3 !} \frac{2 \kappa}{\alpha^{\prime}} \mathcal{R}^{16 / 3} \frac{2 C_{+,+,+}}{V_{D-2}}\left(t^{3}+\left(t^{*}\right)^{3}\right),
$$

where the factor of 2 in front of $C_{+,+,+}$arises from the ghost correlator. The coupling of bulk and twisted tachyons is given by (4.13), with an extra symmetry factor of two, and the power of $\mathcal{R}$ changed due to the different conformal dimension:

$$
\mathbb{V}_{u t^{*} t}=\frac{4 \kappa}{\alpha^{\prime}} \mathcal{R}^{\frac{50}{9}} t^{*} t \int \frac{d^{2} p}{(2 \pi)^{2}}\left(\mathcal{R}^{2} \delta\right)^{-\frac{1}{4} \alpha^{\prime} p^{2}} u(p), \quad \delta=3^{3} \text { for } \mathbb{C} / \mathbb{Z}_{3}
$$

We have now obtained all the contributions to $\mathbb{V}_{3}$. Using $\xi$ introduced in (4.15), setting $u(\xi)=\frac{4 \kappa}{\alpha^{\prime}} u(p)$, and letting $t \rightarrow \frac{\sqrt{\alpha^{\prime}}}{\kappa} t$, the dimensionless ratio $f_{3}$ can be written as

$$
\begin{aligned}
f_{3}=\frac{3 \kappa^{2} \mathbb{V}_{3}}{4 \pi}= & -\frac{8}{3 \pi} t^{*} t-\frac{3}{8 \pi} \int \frac{d^{2} \xi}{(2 \pi)^{2}} u(-\xi)\left(1-\xi^{2}\right) u(\xi) \\
& +\frac{\beta}{4 \pi}\left(t^{3}+\left(t^{*}\right)^{3}\right)+\frac{3}{\pi} \mathcal{R}^{\frac{50}{9}} t^{*} t \int \frac{d^{2} \xi}{(2 \pi)^{2}}\left(\mathcal{R}^{2} \delta\right)^{-\xi^{2}} u(\xi) \\
& +\frac{1}{8 \pi} \int \prod_{i=1}^{3}\left[\frac{d^{2} \xi_{i}}{(2 \pi)^{2}} \mathcal{R}^{2\left(1-\xi_{i}^{2}\right)} u\left(\xi_{i}\right)\right](2 \pi)^{2} \delta^{(2)}\left(\xi_{1}+\xi_{2}+\xi_{3}\right),
\end{aligned}
$$

where

$$
\beta=2 \sqrt{\alpha^{\prime}} \mathcal{R}^{\frac{16}{3}} \frac{C_{+,+,+}}{V_{D-2}}=\frac{3^{\frac{3}{4}} \mathcal{R}^{\frac{16}{3}}}{\sqrt{2} \pi^{2}} \Gamma^{3}(2 / 3) \simeq 1.63674 .
$$

In coordinate space, $f_{3}$ is given by

$$
\begin{aligned}
f_{3}= & \int_{0}^{\infty} d r r\left(-\frac{3}{4} u(r)\left(\partial^{2}+1\right) u(r)+\frac{3 \mathcal{R}^{\frac{50}{9}} t^{*} t}{2 \pi \ln \left(\mathcal{R}^{2} \delta\right)} e^{-\frac{r^{2}}{4 \ln \left(\mathcal{R}^{2} \delta\right)}} u(r)+\frac{1}{4}\left[\mathcal{R}^{2\left(\partial^{2}+1\right)} u(r)\right]^{3}\right) \\
& -\frac{8}{3 \pi} t^{*} t+\frac{\beta}{4 \pi}\left(t^{3}+\left(t^{*}\right)^{3}\right) .
\end{aligned}
$$

The next excited fields in the twisted sectors have level 8/9 and their kinetic terms have level 16/9. In our present analysis we will only include the twisted tachyon $t$ of level 2/9. Accordingly, we restrict bulk tachyon modes to have level less than or equal to $2 / 9$. This corresponds to the following momentum cutoff:

$$
\ell(u(p))=\frac{\alpha^{\prime} p^{2}}{2}=2 \xi^{2} \leq \frac{2}{9} \quad \rightarrow \quad|\xi| \leq \xi_{*}=\frac{1}{3} .
$$

The resulting interactions are shown in Fig. 4. Interactions that involve the excited twisted tachyons are also shown in this figure, but we do not include them in our analysis. 


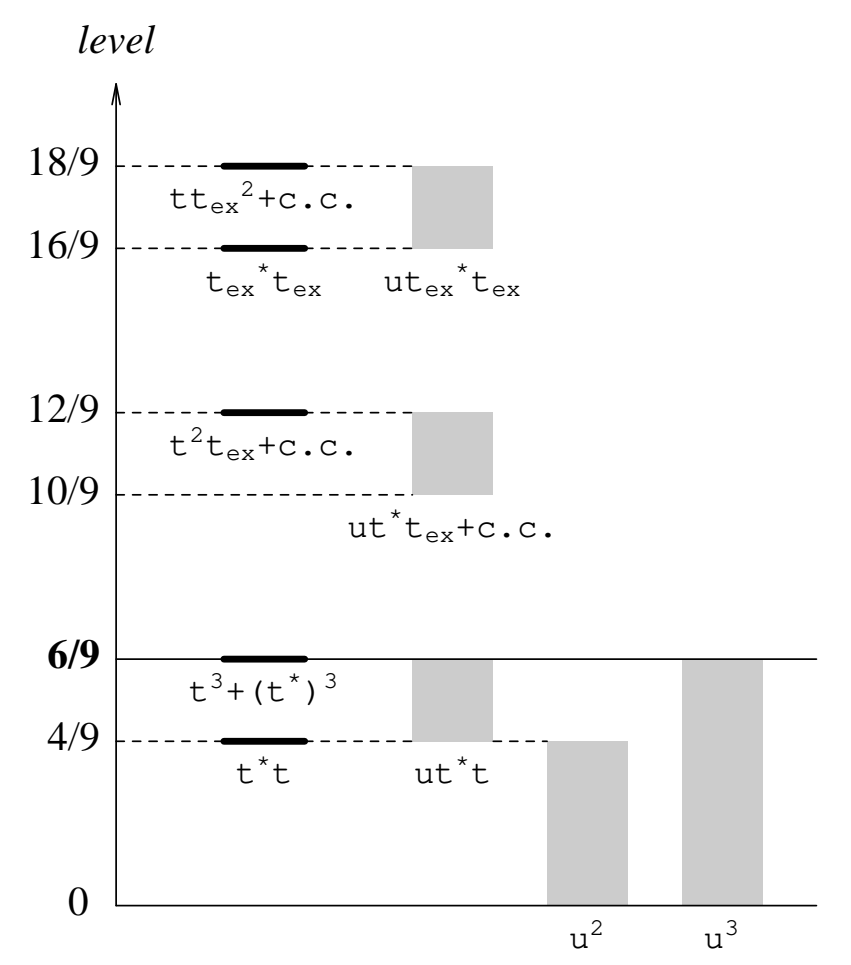

Figure 4: Level expansion table for the $\mathbb{C} / \mathbb{Z}_{3}$ orbifold. Since the bulk tachyon includes all levels from zero to $2 / 9$, interactions that involve the bulk tachyon are shown as bands. Terms involving excited twisted tachyons $t_{\text {ex }}$ are shown, but they are not included in our analysis.

\subsection{A preliminary analysis}

Let us first study $f_{3}$ without the $u^{3}$ term and the $t^{3}+\left(t^{*}\right)^{3}$ term. The equation of motion for $u(\xi)$ can be solved in terms of $t^{*} t$, and by substituting back into $f_{3}$, we find the following effective potential:

$$
f_{3}=\frac{3}{4 \pi}\left[-m_{t^{*} t}^{2} t^{*} t+\frac{\gamma}{2}\left(t^{*} t\right)^{2}\right], \quad m_{t^{*} t}^{2}=\frac{32}{9}, \quad \gamma=16 \eta\left(\mathcal{R}, \delta, \xi_{*}\right) \mathcal{R}^{\frac{100}{9}} \simeq 1.83463 .
$$

Since the $U(1)$ symmetry $t \rightarrow e^{i \theta} t$ is unbroken, the potential has a minimum at $|t|=m_{t^{*} t} / \sqrt{\gamma} \simeq$ 1.39213 and

$$
f_{3}=-\frac{3 m_{t^{*} t}^{4}}{8 \pi \gamma} \simeq-0.822524
$$

This is a surprisingly good estimate. The flat direction in the above potential is removed when we introduce back the cubic terms $t^{3}+\left(t^{*}\right)^{3}$ :

$$
f_{3}=\frac{3}{4 \pi}\left[-m_{t^{*} t}^{2} t^{*} t+\frac{\beta}{3}\left(t^{3}+\left(t^{*}\right)^{3}\right)+\frac{\gamma}{2}\left(t^{*} t\right)^{2}\right] .
$$

The $U(1)$ symmetry is broken to $\mathbb{Z}_{3}$, and we find six critical points:

$$
t_{ \pm}=\frac{-\beta \pm \sqrt{\beta^{2}+4 \gamma m_{t^{*} t}^{2}}}{2 \gamma}, \quad e^{2 \pi i / 3} t_{ \pm}, \quad \text { and } \quad e^{4 \pi i / 3} t_{ \pm} .
$$




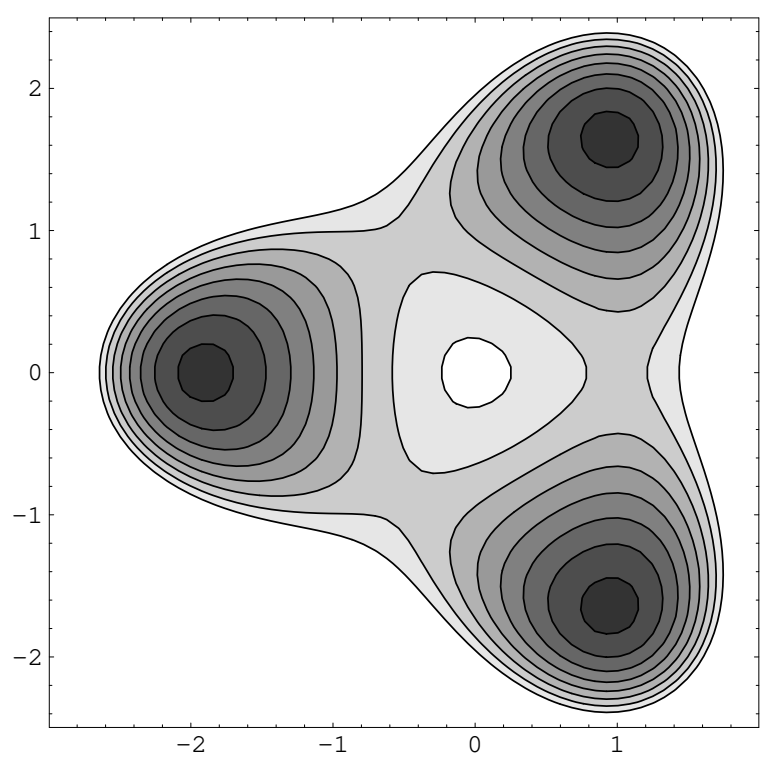

Figure 5: A contour-plot giving the approximate effective potential for the complex tachyon $t$ of the $\mathbb{C} / \mathbb{Z}_{3}$ orbifold. The horizontal and vertical axes correspond to the real and imaginary parts of $t$, respectively. The three saddle points and the three local minima respect the $\mathbb{Z}_{3}$ symmetry.

The critical points $\left(t_{-}, e^{2 \pi i / 3} t_{-}, e^{4 \pi i / 3} t_{-}\right)$are equivalent local minima of the effective potential, while $\left(t_{+}, e^{2 \pi i / 3} t_{+}, e^{4 \pi i / 3} t_{+}\right)$are equivalent saddle points (see Fig. 5 ). Substituting the values of the various parameters, we find

$$
t_{-} \simeq-1.90792, f_{3}\left(t_{-}\right) \simeq-1.99722 ; \quad t_{+} \simeq 1.01578, f_{3}\left(t_{+}\right) \simeq-0.369658
$$

While $f_{3}\left(t_{-}\right)$changed considerably from the earlier estimate in (5.13), it will turn out that $f_{3}\left(t_{-}\right)$ comes back to a value near minus one when we further include the $u^{3}$ terms. This result, as well as the patterns of Fig. 4, suggests that the cubic terms in the twisted and bulk tachyons should be included simultaneously. We claim that the deeper critical point $t_{-}$represents flat space. The shallower critical point $t_{+}$is a natural candidate for the $\mathbb{C} / \mathbb{Z}_{2}$ orbifold. As indicated in (2.12), we expect $f_{3}=-0.25$ at the critical point that corresponds to the $\mathbb{C} / \mathbb{Z}_{2}$ orbifold, and the above result is in fact close to this value. Further evidence for these identifications will arise in the following subsection where we investigate the mass spectrum around the critical points of the full potential.

\subsection{Level expansion analysis}

Let us now study $f_{3}$ including the $u^{3}$ term. It turns out that the cubic interaction does not change the qualitative nature of the critical points we obtained based on (5.14). We have carried out the level-truncation analysis numerically using the coordinate space method in \$4.3.1. As in the case of $\mathbb{C} / \mathbb{Z}_{2}$, we found three branches of solutions where $\left|f_{3}\right|$ remains small as we increase $n$ : one with $t<0$, one with $t>0$, and one with $t=0$. The solution with a vanishing $t$ is not relevant to our current interest. The solutions with $t>0$ and $t<0$ are not equivalent, and they correspond to $t_{+}$and $t_{-}$in 
the previous subsection, respectively. Using the data for $n=16,17, \ldots, 30$, the values of $f_{3}$ can be fitted as follows:

$$
\begin{aligned}
& f_{3}=-0.988865+\frac{1}{n} 0.886950 \text { for the branch with } t<0, \\
& f_{3}=-0.335624+\frac{1}{n} 0.159658 \text { for the branch with } t>0 .
\end{aligned}
$$

For a given $n$, the full potential is a function of $n+2$ real modes: $n$ from the bulk tachyon and two from the twisted tachyon. We have studied the mass spectrum around the critical points. At the critical point with $t<0$, two modes become massive and $n$ modes remain tachyonic. This is in fact the structure of flat space with $n$ bulk tachyon modes included. This provides further evidence that the critical point with $t<0$ represents flat space. At the critical point with $t>0$, only one mode becomes massive and $n+1$ modes remain tachyonic. This is in fact the tachyon structure of the $\mathbb{C} / \mathbb{Z}_{2}$ orbifold with $n$ bulk tachyon modes included. It is therefore tempting to identify the critical point with $t>0$ as the $\mathbb{C} / \mathbb{Z}_{2}$ orbifold.

Our level-truncation result in (5.17) gives about $99 \%$ of the predicted value for the depth of the critical point that represents flat space. This accuracy is clearly accidental. For the critical point argued to represent the $\mathbb{C} / \mathbb{Z}_{2}$ orbifold, the value $f_{3} \simeq-0.34$ obtained from (5.18) is within reasonable range of the expected value $f_{3}=-0.25$. It represents about $134 \%$ of the expected depth. In the same way as we discussed at the end of 4.3 .1 we can attempt to raise the level of the bulk tachyon modes without conflicting with the rules of level expansion. Assume we allow interactions with level up to 9/9, a value slightly below the level of interactions involving new fields in Fig. 4. Then the maximum level allowed for tachyon modes is $1 / 3$, which corresponds to $\xi_{*}=\sqrt{1 / 6}$. For this cutoff value we find

$$
\begin{aligned}
& f_{3}=-0.691264+\frac{1}{n} 0.548867, \quad \text { for the branch with } t<0, \\
& f_{3}=-0.277749+\frac{1}{n} 0.119452, \quad \text { for the branch with } t>0 .
\end{aligned}
$$

As in the case of $\mathbb{C} / \mathbb{Z}_{2}$, the vacua have become shallower, but the values are still good. It would be interesting to improve our analysis by including higher-level fields and interactions.

\section{$6 \quad$ Elementary four-point vertices}

In closed string field theory the quartic term $\langle\Psi, \Psi, \Psi, \Psi\rangle$ is an integral of a suitable measure over some specific subspace $\mathcal{V}_{0,4}$ of the moduli space $\mathcal{M}_{0,4}$ of four-punctured spheres. Each Riemann surface in $\mathcal{V}_{0,4}$ is nicely presented by attaching a semi-infinite cylinder of circumference $2 \pi$ to each of the faces of a tetrahedron. The various surfaces arise by varying the lengths of the edges of the tetrahedron, subject to certain conditions. Explicit computations require the (very nontrivial) conformal map of such constructed surface to the standard presentation as the Riemann sphere $\widehat{\mathbb{C}}$ punctured at $0,1, x$ and $\infty$. The (complex) value of $x$ depends on the parameters of the tetrahedron. The images of the semi-infinite cylinders divide $\widehat{\mathbb{C}}$ into four one-punctured disks. For operators that are matter primaries times $c \bar{c}$, the geometrical information needed to compute the corresponding four-string vertex is (i) 
the four mapping radii $\rho_{1}, \rho_{2}, \rho_{3}$, and $\rho_{4}$ as a function of the modular parameter $x$ and (ii) the region $\mathcal{D}$ such that $x \in \mathcal{D}$ gives all the surfaces in $\mathcal{V}_{0,4}$. Fortunately, Moeller [35] has been able to calculate both the mapping radii and the domain $\mathcal{D}$ efficiently. So, the numerical evaluation of any four-string interaction that involves only primary operators is now possible.

The measure of integration for the quartic term $\langle\Psi, \Psi, \Psi, \Psi\rangle$ in (2.14) is given by a manifestly positive function of the mapping radii times the CFT correlator of the (matter) operators in question, times a minus sign. This minus sign is a nontrivial fact about bosonic closed string field theory (the sign of the CFT correlator is unambiguous because it is related by factorization to products of three-point functions). For bulk tachyons the CFT correlator is manifestly positive and therefore the resulting quartic term in the potential (minus the action) is negative definite and tends to destabilize the vacuum. Indeed, the elementary quartic term overwhelms the cubic term, so the critical point of the cubic potential disappears [20, 21].

It is natural to ask if the same fate awaits the potential calculated in the present paper. In fact, the CFT correlator $Z(x, \bar{x})$ of four twist fields is also manifestly positive, so the quartic term of the twisted tachyons will tend to destabilize the vacuum as well. As we mentioned earlier, we suspect that four-string interactions carry an intrinsic level, perhaps of value near one. If this is the case, the inclusion of the various four-string elementary vertices will typically be accompanied with the inclusion of other interactions that involve excited tachyons of the twisted sector. These latter interactions, just like those of the bulk tachyon, tend to induce stabilizing terms that may possibly compensate for the destabilizing effects of the four-string interactions. The full calculation should be done, but it is nontrivial. Here we do a simple test: we calculate the elementary quartic term of the twisted tachyon and determine if it, alone, destabilizes the vacuum found in the previous sections. We find that it does not.

We consider, for definiteness, the case of the $\mathbb{C} / \mathbb{Z}_{3}$ orbifold. With the string field (5.2), the quartic contribution to the potential is

$$
\begin{aligned}
\mathbb{V}_{\left(t^{*} t\right)^{2}} & =\frac{2}{\alpha^{\prime}} \cdot \frac{1}{4 !} \cdot \frac{\kappa^{2}}{V_{D-2}}\langle\Psi, \Psi, \Psi, \Psi\rangle \\
& =\frac{2}{\alpha^{\prime}} \frac{1}{4 !} \frac{i}{2 \pi} \cdot(2 i) \cdot 2 \cdot 6\left(t^{*} t\right)^{2} \cdot \frac{\kappa^{2}}{V_{D-2}} \int_{\mathcal{D}} d u d v \frac{Z(x, \bar{x})}{\left(\rho_{1} \rho_{2} \rho_{3} \rho_{4}\right)^{2-\frac{2}{9}}},
\end{aligned}
$$

where $x=u+i v$. The factor $(i /(2 \pi))$ is part of the definition of the four-string vertex and the $2 i$ arises from antighost insertions; their product gives the sign mentioned above. The factor of 2 is from the basic overlap and the factor of 6 arises because the product of four string fields contains six terms with two $\sigma_{+}$'s and two $\sigma_{-}$'s. As expected, each mapping radius appears with the power of two minus twice the (matter) dimension of the operator. We then write

$$
\mathbb{V}_{\left(t^{*} t\right)^{2}}=-\frac{\kappa^{2}}{2 \alpha^{\prime}}\left(t^{*} t\right)^{2} \frac{2}{\pi} \cdot \int_{\mathcal{D}} \frac{d u d v}{\left(\rho_{1} \rho_{2} \rho_{3} \rho_{4}\right)^{2}}\left[\frac{Z(x, \bar{x})}{V_{D-2}} \cdot\left(\rho_{1} \rho_{2} \rho_{3} \rho_{4}\right)^{\frac{2}{9}}\right] .
$$

Making use of (3.24) we find

$$
\mathbb{V}_{\left(t^{*} t\right)^{2}}=-\frac{\kappa^{2}}{\alpha^{\prime 2}} \frac{\sqrt{3}}{4 \pi^{2}}\left(t^{*} t\right)^{2} \frac{2}{\pi} \cdot \int_{\mathcal{D}} \frac{d u d v}{\left(\rho_{1} \rho_{2} \rho_{3} \rho_{4}\right)^{2}}\left[\frac{|x(1-x)|^{-\frac{4}{9}}\left(\rho_{1} \rho_{2} \rho_{3} \rho_{4}\right)^{\frac{2}{9}}}{F(x) F(1-\bar{x})+F(1-x) F(\bar{x})}\right] .
$$


The integral

$$
\frac{2}{\pi} \cdot \int_{\mathcal{D}} \frac{d u d v}{\left(\rho_{1} \rho_{2} \rho_{3} \rho_{4}\right)^{2}} \simeq 72.39
$$

was evaluated in order to obtain the quartic term in the bulk tachyon potential 21, 35. Interestingly, this information is sufficient to obtain a reasonable estimate for the integral in (6.3). The expression inside brackets in (6.3) turns out to be nearly constant over the full region $\mathcal{D}$; we found this by computing its value at special points on $\mathcal{D}$ where the mapping radii are known. The factor in brackets seems to vary from about 0.372 to about 0.390 (about a $5 \%$ variation). With the data provided by Moeller, we have actually found that the bracket introduces an effective factor of 0.38002 :

$$
\mathbb{V}_{\left(t^{*} t\right)^{2}} \simeq-\frac{\kappa^{2}}{{\alpha^{\prime}}^{2}}\left(t^{*} t\right)^{2} \frac{\sqrt{3}}{4 \pi^{2}} \cdot 72.39 \cdot 0.38002 \simeq-\frac{\kappa^{2}}{{\alpha^{\prime}}^{2}} \cdot 1.20693 \cdot\left(t^{*} t\right)^{2}
$$

The contribution to $f_{3}$ is approximately $-0.28813\left(t^{*} t\right)^{2}$. The critical points of the twisted sector potential $\mathbb{V}_{t^{*} t}+\mathbb{V}_{t^{3}+\left(t^{*}\right)^{3}}$ in fact disappear when $\mathbb{V}_{\left(t^{*} t\right)^{2}}$ is included. The critical points of the full tachyon potential, however, survive the inclusion of this destabilizing interaction term. Indeed, repeating the analysis in $\$ 5.3$ (for $n=30$ ) with this additional quartic term gives $f_{3} \simeq-1.32$ for the vacuum that represents flat space and $f_{3} \simeq-0.71$ for the vacuum that represents the $\mathbb{C} / \mathbb{Z}_{2}$ orbifold. The elementary quartic interaction $\mathbb{V}_{\left(t^{*} t\right)^{2}}$ decreases $f_{3}$, as expected. We have seen at the end of $\$ 5.3$ that higher-momentum modes of the bulk tachyon increase $f_{3}$. There are other interactions involving fields at the next excited level which are expected to affect $f_{3}$ at the same order. It would be interesting and important to include these interactions in our analysis to see if the critical points survive and the values of $f_{3}$ improve.

\section{Conclusions and discussion}

The computations we have done in this paper provide new evidence for the conjecture of Adams, Polchinski, and Silverstein [1] (APS). The potentials we obtain for low-level tachyons in the $\mathbb{C} / \mathbb{Z}_{2}$ and $\mathbb{C} / \mathbb{Z}_{3}$ orbifolds have the qualitative and quantitative features required for tachyon condensation to trigger background changes to orbifolds with smaller deficit angles or to flat space. In our low-level calculations the metric is not dynamical and the conjecture of Dabholkar [15] can be tested. We find evidence that the tachyon potentials have critical points at depths that roughly generate the expected changes in deficit angles. Our calculations were done in the framework of closed string field theory, demonstrating that this theory can be used to study nonperturbative closed string physics.

We have found that the physically relevant potential depends on twisted fields and localized bulk field excitations. For computations that reach level two - the level of massless closed string fieldsthe metric dependence of $\mathbb{V}$ becomes explicit, as we have to include the gravitational field into the computations. We have argued that for a metric dependent potential the conjecture [15] may not hold exactly. The metric dependence does not arise solely from the localized bulk field excitations. The Gaussian profile of the CFT three-point function (3.19) shows that twisted fields are not $\delta$-functionally

localized but rather smeared out in a scale of order $\sqrt{\alpha^{\prime}}$. Their couplings to bulk fields thus contain 
form factors that represent unusual metric dependence. A calculation that includes gravity would be of level $(2,6)$ and the typical level of our present computations is $(1 / 4,3 / 4)$. We have some way to go before we can discuss the effects of massless fields quantitatively.

In any computation beyond level two one must include the equation of motion for gravity. Once the gravitational field is allowed to condense, the APS conjecture cannot be tested by looking at the change in the action, as we did in this paper. Indeed, we saw in 2.1 that the action vanishes at the classical solutions. It is not clear to us whether there is a version of the Dabholkar conjecture that holds once the gravitational back-reaction is included. To find a way to test the APS conjecture beyond level two is an important future problem. One possibility may be to read off the deficit angle of the spacetime from the metric that satisfies the string field equations. This may be challenging in the present form of the string field theory action where the direct identification of the graviton field with the metric fluctuation is valid only for small fields. Another possibility might entail the examination of boundary contributions to the gravitational action.

There are also a host of technical questions. Additional work is necessary in order to demonstrate that closed string field theory can be used in some suitably defined level expansion. There may also be other closed string backgrounds that can be used to test the level expansion 36, 37. The rules of closed string theory level expansion should be found. The present calculations may be carried out to higher levels, including systematically the effects of (at least) quartic terms. Since there are both positive and negative contributions to the potential, it is important to verify that the critical points survive and the ratios $f_{N}$ at the critical points become closer to the predicted values.

It would be interesting to extend our analysis of tachyonic instabilities to the $\mathbb{R} / \mathbb{Z}_{2}$ orbifold, which is not a cone but rather a half-line. There is no prediction here for final state and no conjecture concerning the critical points of the tachyon potential. It is also of interest to analyze orbifolds of finite volume, in which case the end product of the decay does not appear to be known either. Finally, it would be very useful to develop a workable closed superstring field theory of the NS-NS sector. We could then test the original version of the conjectures directly in the context of Type II backgrounds that have no bulk tachyon. It would be interesting to see how the critical points of the twisted tachyon potentials are generated.

\section{Acknowledgements}

We would like to thank A. Adams, O. Bergman, A. Dabholkar, M. Headrick, R. Jackiw, D. Kutasov, H. Liu, S. Minwalla, N. Moeller, J. Raeymaekers, M. Schnabl, A. Sen, W. Taylor, and C. Vafa for useful discussions. We are grateful to the authors of [17] and [18 for discussing their results prior to

publication. We also thank N. Moeller for providing the data necessary for the evaluation of four-string interactions. B.Z. thanks the kind hospitality of Harvard University, where part of this work was done. This work was supported in part by the DOE grant DF-FC02-94ER40818. 


\section{References}

[1] A. Adams, J. Polchinski and E. Silverstein, "Don't panic! Closed string tachyons in ALE space-times," JHEP 0110, 029 (2001) arXiv:hep-th/0108075.

[2] C. Vafa, "Mirror symmetry and closed string tachyon condensation," arXiv:hep-th/0111051

[3] J. A. Harvey, D. Kutasov, E. J. Martinec and G. Moore, "Localized tachyons and RG flows," arXiv:hep-th/0111154

[4] R. Gregory and J. A. Harvey, "Spacetime decay of cones at strong coupling," Class. Quant. Grav. 20, L231 (2003) arXiv:hep-th/0306146.

[5] M. Headrick, "Decay of C/Z(n): Exact supergravity solutions," arXiv:hep-th/0312213.

[6] A. Dabholkar, "Strings on a cone and black hole entropy," Nucl. Phys. B 439, 650 (1995) arXiv:hep-th/9408098; D. A. Lowe and A. Strominger, "Strings near a Rindler or black hole horizon," Phys. Rev. D 51, 1793 (1995) arXiv:hep-th/9410215.

[7] A. Dabholkar, "On condensation of closed-string tachyons," Nucl. Phys. B 639, 331 (2002) arXiv:hep-th/0109019; A. Dabholkar and C. Vafa, "tt* geometry and closed string tachyon potential," JHEP 0202, 008 (2002) arXiv:hep-th/0111155.

[8] J. R. David, M. Gutperle, M. Headrick and S. Minwalla, "Closed string tachyon condensation on twisted circles," JHEP 0202, 041 (2002) arXiv:hep-th/0111212; M. Gutperle, M. Headrick, S. Minwalla and V. Schomerus, "Space-time energy decreases under world-sheet RG flow," JHEP 0301, 073 (2003) arXiv:hep-th/0211063.

[9] S. P. De Alwis and A. T. Flournoy, "Closed string tachyons and semi-classical instabilities," Phys. Rev. D 66, 026005 (2002) arXiv:hep-th/0201185; S. J. Sin, "Tachyon mass, c-function and counting localized degrees of freedom," Nucl. Phys. B 637, 395 (2002) arXiv:hep-th/0202097; A. Basu, "Localized tachyons and the $\mathrm{g}(\mathrm{cl})$ conjecture," JHEP 0207, 011 (2002) arXiv:hep-th/0204247; T. Sarkar, "Brane probes, toric geometry, and closed string tachyons," Nucl. Phys. B 648, 497 (2003) arXiv:hep-th/0206109; J. R. David, "Unstable magnetic fluxes in heterotic string theory," JHEP 0209, 006 (2002) arXiv:hep-th/0208011; T. Suyama, "Closed string tachyons and RG flows," JHEP 0210, 051 (2002) arXiv:hep-th/0210054; E. J. Martinec, "Defects, decay, and dissipated states," arXiv:hep-th/0210231 A. Armoni, E. Lopez and A. M. Uranga, "Closed strings tachyons and non-commutative instabilities," JHEP 0302, 020 (2003) arXiv:hep-th/0301099; Y. H. He, "Closed string tachyons, non-supersymmetric orbifolds and generalised McKay correspondence," Adv. Theor. Math. Phys. 7, 121 (2003) arXiv:hep-th/0301162; M. Dine, E. Gorbatov, I. R. Klebanov and M. Krasnitz, "Closed string tachyons and their implications for nonsupersymmetric strings," arXiv:hep-th/0303076 B. C. Da Cunha and E. J. Martinec, "Closed string tachyon condensation and worldsheet inflation," Phys. Rev. D 68, 063502 (2003) arXiv:hep-th/0303087; S. Minwalla and T. Takayanagi, "Evolution of D-branes under closed string tachyon condensation," JHEP 0309, 011 (2003) arXiv:hep-th/0307248; S. J. Sin, "Comments on the fate of unstable orbifolds," Phys. Lett. B 578, 215 (2004) arXiv:hep-th/0308028; S. Groot Nibbelink and M. Laidlaw, "Stringy profiles of gauge field tadpoles near orbifold singularities. I: Heterotic string calculations," JHEP 0401, 004 (2004) arXiv:hep-th/0311013; S. Lee and S. J. Sin, "Localized tachyon condensation and G-parity conservation," arXiv:hep-th/0312175 G. Moore and A. Parnachev, "Localized Tachyons and the Quantum McKay Correspondence," arXiv:hep-th/0403016

[10] B. Zwiebach, "Closed string field theory: Quantum action and the B-V master equation," Nucl. Phys. B 390, 33 (1993) hep-th/9206084.

[11] M. Saadi and B. Zwiebach, "Closed String Field Theory From Polyhedra," Annals Phys. 192, 213 (1989). 
[12] T. Kugo and K. Suehiro, "Nonpolynomial Closed String Field Theory: Action And Its Gauge Invariance," Nucl. Phys. B 337, 434 (1990); T. Kugo, H. Kunitomo and K. Suehiro, "Nonpolynomial Closed String Field Theory," Phys. Lett. B 226, 48 (1989).

[13] M. Kaku and J. Lykken, "Modular Invariant Closed String Field Theory," Phys. Rev. D 38, 3067 (1988); M. Kaku, "Geometric Derivation Of String Field Theory From First Principles: Closed Strings And Modular Invariance," Phys. Rev. D 38, 3052 (1988).

[14] N. Moeller, A. Sen and B. Zwiebach, "D-branes as tachyon lumps in string field theory," JHEP 0008, 039 (2000) arXiv:hep-th/0005036.

[15] A. Dabholkar, "Tachyon condensation and black hole entropy," Phys. Rev. Lett. 88, 091301 (2002) arXiv:hep-th/0111004.

[16] S. Sarkar and B. Sathiapalan, "Closed string tachyons on C/Z(N)," arXiv:hep-th/0309029

[17] A. Dabholkar, A. Iqubal and J. Raeymaekers, to appear.

[18] A. Adams, A. Dabholkar, M. Headrick and J. Raeymaekers, work in progress.

[19] A. Sen and B. Zwiebach, "Tachyon condensation in string field theory," JHEP 0003, 002 (2000) arXiv:hep-th/9912249.

[20] A. Belopolsky and B. Zwiebach, "Off-shell closed string amplitudes: Towards a computation of the tachyon potential," Nucl. Phys. B 442, 494 (1995) arXiv:hep-th/9409015.

[21] A. Belopolsky, "Effective Tachyonic potential in closed string field theory," Nucl. Phys. B 448, 245 (1995) arXiv:hep-th/9412106.

[22] D. Gaiotto and L. Rastelli, "Experimental string field theory," JHEP 0308, 048 (2003) arXiv:hep-th/0211012.

[23] W. Taylor, "A perturbative analysis of tachyon condensation," JHEP 0303, 029 (2003) arXiv:hep-th/0208149.

[24] A. Vilenkin, "Gravitational Field Of Vacuum Domain Walls And Strings," Phys. Rev. D 23, 852 (1981).

[25] A. Vilenkin, "Cosmic Strings And Domain Walls," Phys. Rept. 121, 263 (1985).

[26] S. Deser, R. Jackiw and G. 't Hooft, "Three-Dimensional Einstein Gravity: Dynamics Of Flat Space," Annals Phys. 152, 220 (1984).

[27] S. W. Hawking and G. T. Horowitz, "The Gravitational Hamiltonian, action, entropy and surface terms," Class. Quant. Grav. 13, 1487 (1996) arXiv:gr-qc/9501014.

[28] J. Polchinski, String Theory, Vol. I: An introduction to the bosonic string, Cambridge University Press, UK (1998).

[29] V. A. Kostelecky and S. Samuel, "Collective Physics In The Closed Bosonic String," Phys. Rev. D 42, 1289 (1990).

[30] O. Bergman, unpublished.

[31] L. J. Dixon, D. Friedan, E. J. Martinec and S. H. Shenker, "The Conformal Field Theory Of Orbifolds," Nucl. Phys. B 282, 13 (1987).

[32] S. Hamidi and C. Vafa, "Interactions On Orbifolds," Nucl. Phys. B 279, 465 (1987). 
[33] M. A. Bershadsky and A. O. Radul, "Conformal Field Theories With Additional Z(N) Symmetry," Sov. J. Nucl. Phys. 47 (1988) 363 [Yad. Fiz. 47 (1988) 575].

[34] P. Kraus, A. Ryzhov and M. Shigemori, "Strings in noncompact spacetimes: Boundary terms and conserved charges," Phys. Rev. D 66, 106001 (2002) arXiv:hep-th/0206080.

[35] N. Moeller, to appear.

[36] S. Mukherji and A. Sen, "Some all order classical solutions in nonpolynomial closed string field theory," Nucl. Phys. B 363, 639 (1991); S. Mukherji, S. Mukhi and A. Sen, "Black hole solution and its infinite parameter generalizations in $c=1$ string field theory," Phys. Lett. B 275, 39 (1992); A. Sen, "Some applications of string field theory," arXiv:hep-th/9109022

[37] A. Sen and B. Zwiebach, "A Proof of local background independence of classical closed string field theory," Nucl. Phys. B 414, 649 (1994) arXiv:hep-th/9307088. 